\title{
MÉTODO SIMPLIFICADO DE EVALUACIÓN SÍSMICA DE EDIFICIOS ASIMÉTRICOS
}

\author{
A. Gustavo Ayala ${ }^{(1)}$, Elías A. Tavera ${ }^{(1)}$ y Mauricio Ayala ${ }^{(2)}$
}

\begin{abstract}
RESUMEN
En este artículo se presenta un método aproximado de evaluación del comportamiento sísmico de edificios asimétricos. El método se basa en un análisis no-lineal de empujón, en la reducción de la curva de capacidad a una de comportamiento de un sistema equivalente de un grado de libertad, en el cálculo de su respuesta máxima ante una demanda sísmica considerada y en la transformación de ésta a la correspondiente de la estructura original. En la aplicación del análisis del empujón, la estructura se empuja en dos direcciones ortogonales con fuerzas laterales de distribuciones y proporciones como lo recomienda el reglamento vigente. El potencial del método propuesto para estimar el desempeño sísmico de edificios asimétricos se demuestra al comparar los resultados de dos ejemplos ilustrativos, con los correspondientes a los de análisis dinámicos no lineales paso a paso.
\end{abstract}

\section{SUMMARY}

This paper presents an approximate method for the seismic performance evaluation of asymmetric building structures. The method is based on a non-linear pushover analysis, the transformation of the capacity curve into a behavior curve of an equivalent single degree of freedom system, the evaluation of its maximum response to a given seismic demand and the back transformation of the response to the original structure. In the application of the pushover analysis, the structure is pushed in two orthogonal directions with lateral forces with distributions and proportions defined as recommended by the current code. The potentiality of the proposed method to estimate seismic performance of asymmetric buildings is shown when the approximate results for two illustrative examples are compared with those obtained with "true" non-linear step by step analyses.

\section{INTRODUCCIÓN}

En los códigos actuales de diseño sísmico se acepta para el análisis el uso de métodos lineales estáticos o dinámicos; sin embargo, estos tipos de análisis pueden ser insuficientes para describir el comportamiento real de estructuras ante fuerzas dinámicas como las producidas por los sismos intensos que definen las acciones de diseño. Para lograr lo anterior es necesario usar métodos de

Artículo recibido el 10 de octubre de 2001 y aprobado para su publicación el 17 de mayo de 2002. Se aceptarán comentarios y/o discusiones hasta cinco meses después de su publicación.

(1) Instituto de Ingeniería, UNAM, Ciudad Universitaria, 04510 México, DF. gayala@dali.fi-p.unam.mx, elias_antolin98@hotmail.com

(2) Instituto Tecnológico de Sonora, Cd. Obregón, Sonora. mayala@itson.mx 
análisis dinámico no-lineal paso a paso que, para fines prácticos, no representan la opción más recomendable para el análisis sísmico de estructuras tridimensionales (3D) de edificio, ya que son complejos en su concepción y en su uso. Este tipo de análisis sólo se justifica para ciertas aplicaciones donde se requieren resultados refinados, como es el caso de investigaciones y/o proyectos especiales.

Debido a lo anterior, y ante la necesidad de contar con herramientas asequibles a los ingenieros, recientemente se han desarrollado varios procedimientos simplificados de análisis nolineal, para evaluar el comportamiento sísmico de estructuras de edificios en tres dimensiones. La mayoría de estos estudios se basan en análisis del empujón en dos dimensiones en los que la estructura tridimensional se evalúa a partir de las curvas de comportamiento de los marcos planos que la forman, por ejemplo Fajfar y Gaspersic (1996). De la variedad de trabajos publicados, sólo Moghadam y Tso (1996, 1997 y 1998) extendieron la aplicación del análisis de empujón en dos dimensiones a edificios asimétricos en 3D considerando elementos resistentes sólo en una dirección. Otros métodos, como el propuesto por Kilar y Fajfar (1997), utilizan los macroelementos en su análisis de empujón, donde la idea principal es que con pocos elementos no lineales se represente el desempeño global de la estructura.

En este artículo se propone un método simplificado de análisis para evaluar el comportamiento sísmico no-lineal de edificios en 3D de varios niveles, en el cual se incluye de manera explícita el efecto de la torsión producida por asimetrías en la distribución de masas y/o rigideces en sus pisos. Este método tiene algunas diferencias importantes con respecto a los procedimientos existentes, como son el empujar la estructura del edificio simultáneamente en dos direcciones ortogonales en consistencia con la acción sísmica, considerar que las distribuciones de las cargas laterales estáticas equivalentes a las sísmicas son las correspondientes a los modos fundamentales laterales de vibrar instantáneos de la estructura y la de fundamentarse en todo momento en conceptos establecidos de la dinámica estructural que permiten reducir a la estructura original a un sistema equivalente de 1GDL correspondiente al modo fundamental de la estructura, Freeman et al. (1975).

Para ilustrar el método propuesto se estudian dos edificios, uno de cuatro y otro de ocho niveles previamente diseñados por Luaces (1995). Para estos diseños se consideran tres valores distintos de excentricidad distribuidos uniformemente en los pisos. Como parámetros de respuesta se consideran los valores máximos de desplazamientos laterales, desplazamientos de entrepiso y la distribución y magnitudes de las articulaciones en los que se concentra el daño estructural.

La buena aproximación de los resultados obtenidos al emplear el método simplificado de este artículo se demuestra al compararlos con los obtenidos de un método riguroso de análisis sísmico no-lineal paso a paso. 


\section{MÉTODO PROPUESTO}

El procedimiento del método propuesto para evaluar estructuras asimétricas tridimensionales consiste en:

1. Determinar la distribución de las fuerzas laterales equivalentes considerando como hipótesis que la estructura vibra dominantemente en sus modos laterales desacoplados correspondientes a los fundamentales en cada dirección de análisis y que las distribuciones de las fuerzas sísmicas equivalentes no cambian significativamente cada vez que ocurran incursiones en el intervalo no-lineal de comportamiento, Requena (1999). Para calcular las distribuciones de fuerzas laterales equivalentes es necesario efectuar un análisis de modos y frecuencias propias de la estructura. La ecuación que representa estas distribuciones de fuerzas laterales para cada dirección de análisis se deriva de conceptos básicos de la dinámica estructural que las definen como, Chopra (2001):

$$
F_{i}=\frac{M_{i} \phi_{i}}{\sum_{j}^{N} M_{j} \phi_{j}} V
$$

donde:

$\mathrm{F}_{\mathrm{i}}=$ fuerza lateral en el nivel $\mathrm{i}$

$\mathrm{M}_{\mathrm{i}}=$ masa del nivel i

$\phi_{\mathrm{i}}=$ ordenada de la forma modal fundamental en el nivel $\mathrm{i}$

$\mathrm{V}=$ cortante basal

$\mathrm{N}=$ número de niveles

La combinación de efectos bidireccionales se hace de acuerdo con lo que establece el RCDF-93 (DDF, 1993), es decir, los efectos de ambas componentes horizontales se combinan para cada dirección principal en que se debe analizar la estructura, 100\% de los efectos de la componente que actúe en esa dirección y 30\% de los efectos en la dirección perpendicular a ella.

2. Realizar de acuerdo con Freeman et al. (1975) un análisis estático no-lineal del empujón en 3D en el que, con un programa de análisis no lineal como el CANNY-E, Li (1996), la estructura se someta a las distribuciones de cargas laterales obtenidas en el paso anterior, incrementando simultáneamente y de manera monótona su magnitud hasta que ésta alcance un estado límite predefinido, generalmente dado por un desplazamiento máximo de azotea. Con los resultados de este análisis se construye la curva de capacidad de la estructura, en la cual se representan los desplazamientos en la dirección de evaluación (aquella en la que se aplica 100\% de la demanda) de un punto característico de la estructura, en este caso seleccionado como el centro de masas del nivel de azotea de la estructura con los valores correspondientes del cortante basal en esa misma dirección.

3. La curva cortante basal contra desplazamiento de azotea que se obtiene en el paso anterior se convierte en una de seudo-aceleración espectral $\left(\mathrm{S}_{\mathrm{a}}\right)$ contra desplazamiento espectral 
$\left(\mathrm{S}_{\mathrm{d}}\right)$ de un sistema equivalente de 1GDL, usando ecuaciones establecidas de la dinámica estructural, Chopra (2001). La curva cortante basal modal contra desplazamiento espectral representa la curva de comportamiento del sistema equivalente de 1GDL. Ya que en general esta curva es multilineal, es conveniente simplificarla aproximándola con una bilineal equivalente, Qi y Moehle (1991), usando recursivamente un sistema que tenga la misma área bajo la curva para los valores máximos obtenidos de un programa de análisis no-lineal de sistemas de 1GDL como el NONLIN, Charney (1998). Para convertir el sistema de VGDL a un sistema equivalente de 1GDL se utilizan las ecuaciones de la dinámica estructural descritas en el ATC-40 (1996) y usadas por Requena (1999):

Seudo-aceleración espectral del sistema de 1GDL

$\mathrm{S}_{\mathrm{a}}=\frac{\mathrm{V}}{\alpha \mathrm{W}}$

Desplazamiento espectral del sistema de 1GDL

$\mathrm{S}_{\mathrm{d}}=\frac{\mathrm{D}}{\mathrm{PF}_{\mathrm{m}}}$

donde:

$\mathrm{PF}_{\mathrm{m}}=\left[\frac{\sum_{\mathrm{i}=1}^{\mathrm{N}}\left(\mathrm{M}_{\mathrm{i}} \phi_{\mathrm{i}}\right)}{\sum_{\mathrm{i}=1}^{\mathrm{N}}\left(\mathrm{M}_{\mathrm{i}} \phi_{\mathrm{i}}^{2}\right)}\right] \phi_{\mathrm{m}}$

$\alpha=\frac{\left[\sum_{i=1}^{N}\left(M_{i} \phi_{i}\right)\right]^{2}}{\left[\sum_{i=1}^{N} M_{i}\right] \sum_{i=1}^{N}\left(M_{i} \phi_{i}^{2}\right)}$

$\mathrm{W}=$ peso total del edificio

$\mathrm{PF}_{\mathrm{m}}=$ factor de participación modal en la azotea

$\phi_{\mathrm{m}}=$ amplitud del modo fundamental en la azotea

$\alpha=$ factor de participación modal del cortante basal

$\mathrm{D}=$ desplazamiento lateral de la azotea en la dirección de análisis

$\mathrm{S}_{\mathrm{a}}=$ seudo-aceleración espectral

$\mathrm{S}_{\mathrm{d}}=$ desplazamiento espectral 
4. La masa y la relación de amortiguamiento del sistema de 1GDL correspondientes a las del modo fundamental normalizado se definen como:

$\mathrm{m}_{1}=\sum_{\mathrm{i}=1}^{\mathrm{N}} \mathrm{M}_{\mathrm{i}} \phi_{\mathrm{i}}^{2}$

$\xi_{1}=0.05$

5. El sistema equivalente de 1GDL se somete al sismo o sismos seleccionados como demanda sísmica para determinar el desplazamiento espectral máximo absoluto que el sistema equivalente experimenta ante ésta. Este desplazamiento, representado por un punto en la curva de comportamiento conocido como punto de desempeño, se transforma en el desplazamiento de azotea del sistema de varios grados de libertad (VGDL) usando de manera inversa las ecuaciones del paso 3.

6. Conocido el desplazamiento de desempeño en el centro de masa (CM) de la azotea de la estructura en la dirección de evaluación, se realiza un segundo análisis de empujón hasta alcanzar este desplazamiento. Los valores aproximados máximos de cortante basal, desplazamientos de cada nivel, desplazamientos relativos de entrepiso, localización y magnitud de rotaciones de las articulaciones plásticas, elementos mecánicos, etc., se obtienen directamente de los resultados de este análisis

\section{EJEMPLOS ILUSTRATIVOS}

\section{Curva cortante basal contra desplazamiento de azotea}

Para ilustrar la aplicación del método propuesto, se seleccionaron dos edificios, uno de cuatro y otro de ocho niveles con una misma distribución de los elementos estructurales en planta, fig. 1. Estos edificios, formados por marcos planos de concreto reforzado, fueron diseñados por Luaces (1995), de acuerdo al Reglamento de Construcciones para el Distrito Federal y las Normas Técnicas Complementarias (DDF, 1993). Los detalles de la configuración de los edificios y su diseño se presentan en Ayala (1999).

Para estudiar el efecto de la excentricidad sobre la calidad de los resultados obtenidos del método propuesto, los edificios se analizaron como estructuras asimétricas en masa con tres valores de excentricidad, $\mathrm{e}_{\mathrm{s}}=0.0 \mathrm{~b}, 0.1 \mathrm{~b}$ y $0.2 \mathrm{~b}$, donde $\mathrm{b}$ es la dimensión de la planta en el sentido de la excentricidad. En el caso simétrico $\left(\mathrm{e}_{\mathrm{s}}=0.0 \mathrm{~b}\right)$ la distribución de masas se considera uniforme sobre cada nivel, por lo que los centros de masas, los de rigideces y los de resistencias de los niveles coinciden con los centros geométricos de las plantas. Para los casos asimétricos, con excentricidades mayores que cero, la distribución de masas en cada nivel fue tal que sus centros se movieron en ambas direcciones una distancia igual a 0.1 b y $0.2 \mathrm{~b}$ del centro geométrico de cada uno de los niveles de la estructura. 


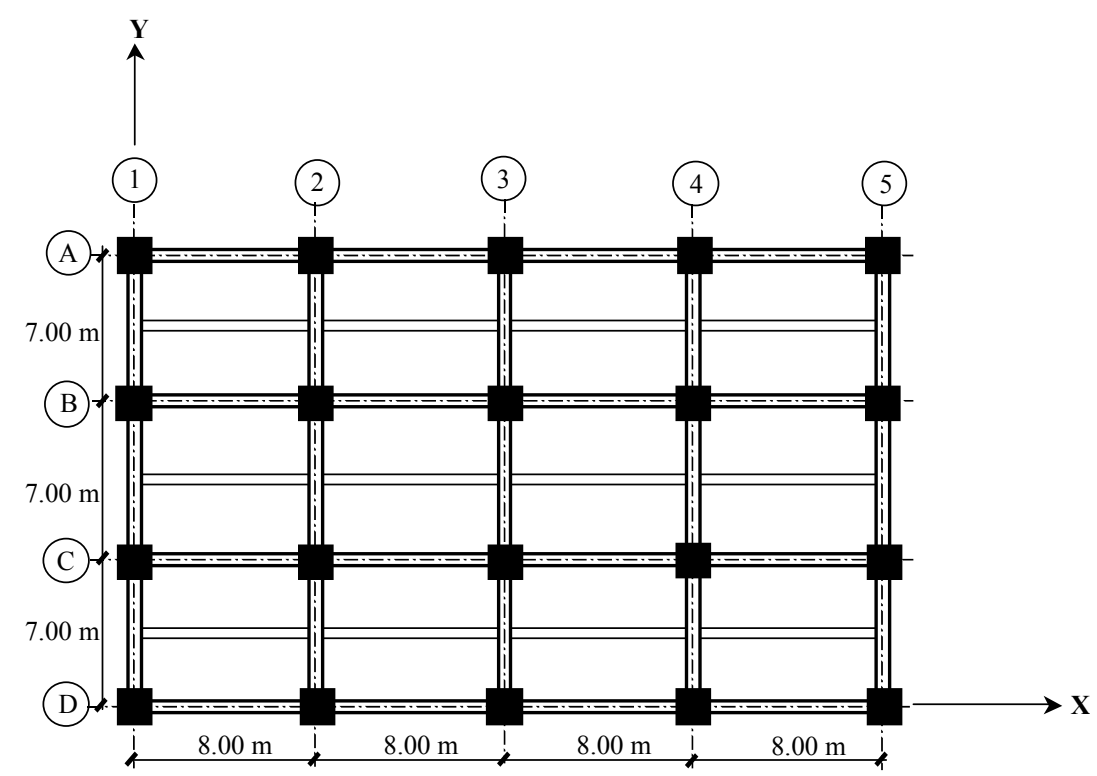

Figura 1. Vista en planta del edificio de cuatro y ocho niveles

Para fines de evaluar el método propuesto, los modelos estructurales se sometieron a análisis dinámicos no-lineales paso a paso considerando para todos los modos una fracción de amortiguamiento crítico $\xi$ del $5 \%$, el cual se consideró apropiado para estructuras de concreto reforzado. Como demanda sísmica se consideraron los registros del sismo del 19 de septiembre de 1985 obtenidos en la estación de la Secretaría de Comunicaciones y Transporte, SCT, en las direcciones este-oeste, SCT E-O, y norte-sur, SCT N-S. Al llevar a cabo los análisis paso a paso de los modelos estructurales las componentes actuaron simultáneamente, sin embargo, en la aplicación del método aproximado, los sistemas de 1GDL sólo se analizan para la componente en la dirección en evaluación, ya que los efectos de la otra componente ya fueron incluidos en las curvas de comportamiento correspondientes a esta dirección.

\section{ANÁLISIS DE RESULTADOS}

En esta sección se discuten y comparan los resultados de análisis realizados con el método aproximado propuesto contra los resultados de análisis dinámicos no-lineales paso a paso. Los análisis del empujón y dinámicos paso a paso en 3D se realizaron con el programa de análisis CANNY-E desarrollado por Li (1996). Los análisis dinámicos del sistema de 1GDL se llevaron a cabo con el programa NONLIN desarrollado por Charney (1998).

\section{PARÁMETROS ESTUDIADOS}

Los parámetros de respuesta que se consideraron de interés para evaluar el método propuesto son: (1) desplazamientos máximos de cada nivel, (2) desplazamientos relativos de entrepiso máximos,

(3) demandas máximas de ductilidad en columnas y trabes. El primer y segundo parámetro 
describen la respuesta global de una estructura y los terceros se utilizan como indicadores del daño estructural para representar la respuesta local de los elementos (Moghadam, 1998).

\section{EDIFICIO DE CUATRO NIVELES}

\section{Curva cortante basal contra desplazamiento de azotea}

En la fig. 2 se muestran las curvas de capacidad (cortante basal contra desplazamiento de azotea) para las tres excentricidades consideradas en el edificio de cuatro niveles. Se observa que en la dirección $\mathrm{X}$ las curvas de capacidad son muy similares para las excentricidades consideradas. Esta casi coincidencia de curvas se debe a una característica particular del ejemplo considerado en el que la distribución de rigideces en las plantas del edificio y las direcciones de la demanda sísmica hacen que los efectos de torsión se contrarresten al sumarse momentos torsionantes de signo diferente. Este efecto no aparece en la dirección de evaluación Y ya que, debido a los sentidos de la demanda sísmica considerada, se observa que al aumentar la excentricidad, la resistencia del edificio disminuye.

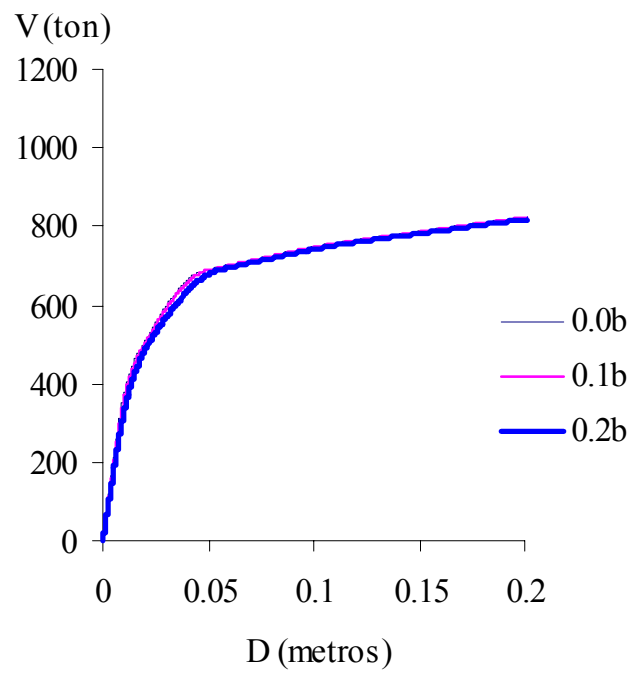

( a ) Dirección X

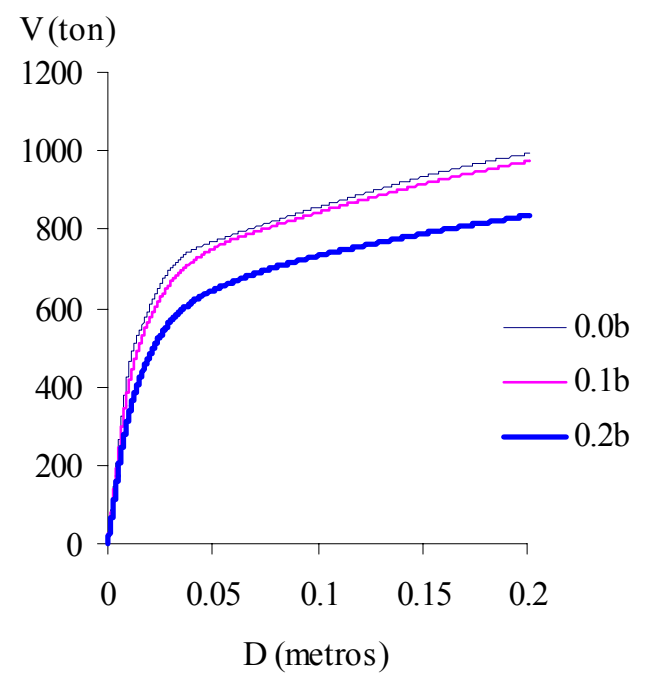

( b ) Dirección Y

Figura 2. Curvas de capacidad para el edificio de cuatro niveles.

\section{Aproximación bilineal de las curvas de empujón}

En la fig. 3 se ilustran las curvas $S_{a}$ contra $S_{d}$ del sistema de 1 GDL correspondiente al CM para los casos considerados y su correspondiente idealización bilineal. Es de importancia mencionar que al no conocerse de antemano el punto de comportamiento, es necesario suponer uno y revisar la validez de esta suposición; de no serlo será necesario repetir este procedimiento hasta que el punto de comportamiento resulte suficientemente cercano al supuesto. Así, en las curvas de 
comportamiento de la fig. 3 sólo se muestran las idealizaciones correspondientes a los puntos de comportamiento obtenidos.

Dirección X

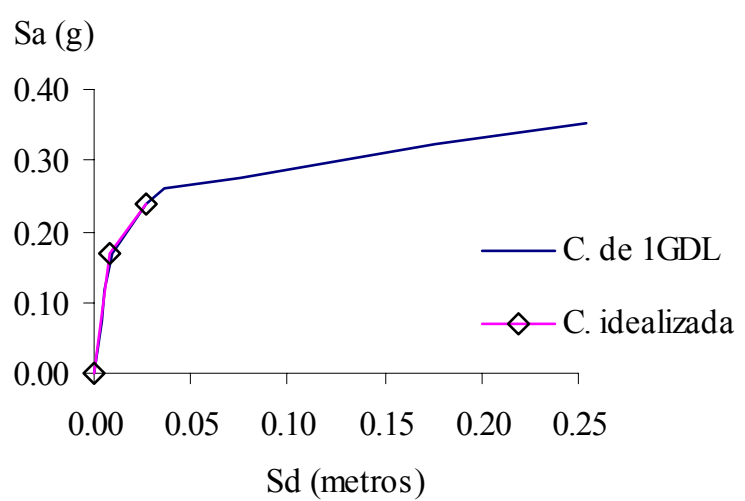

Dirección Y

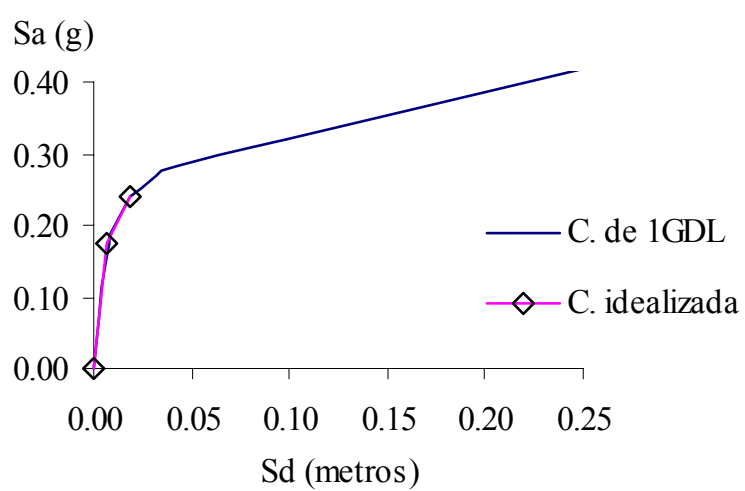

( a ) $\mathrm{e}_{\mathrm{s}}=0.0 \mathrm{~b}$
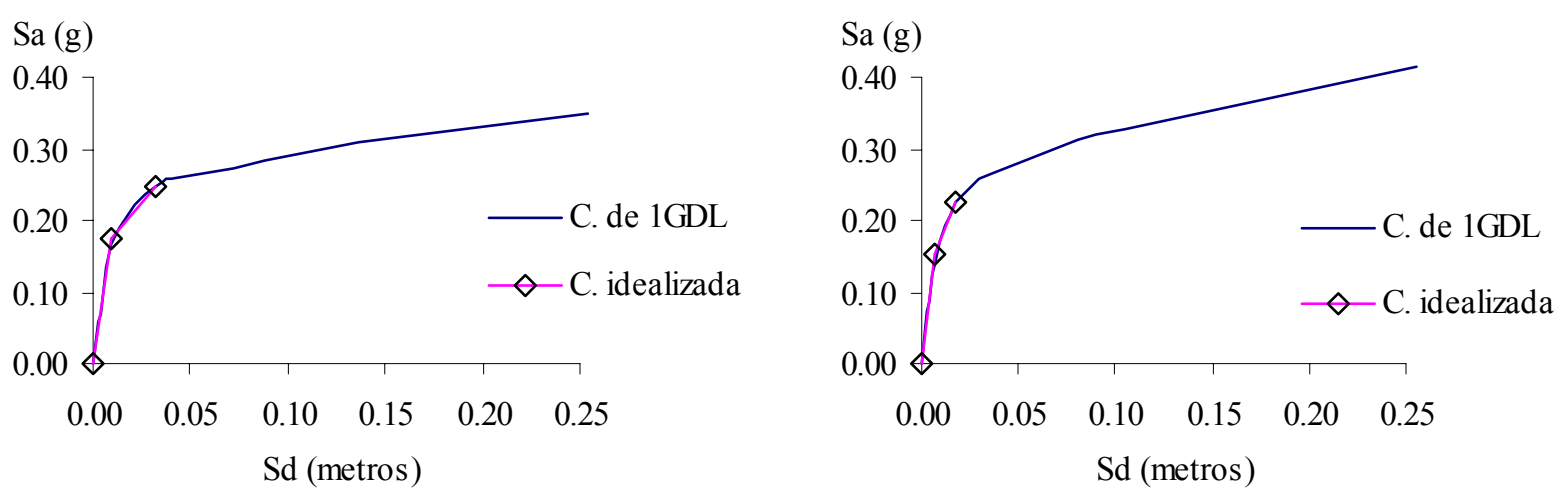

( b ) $\mathrm{e}_{\mathrm{s}}=0.1 \mathrm{~b}$
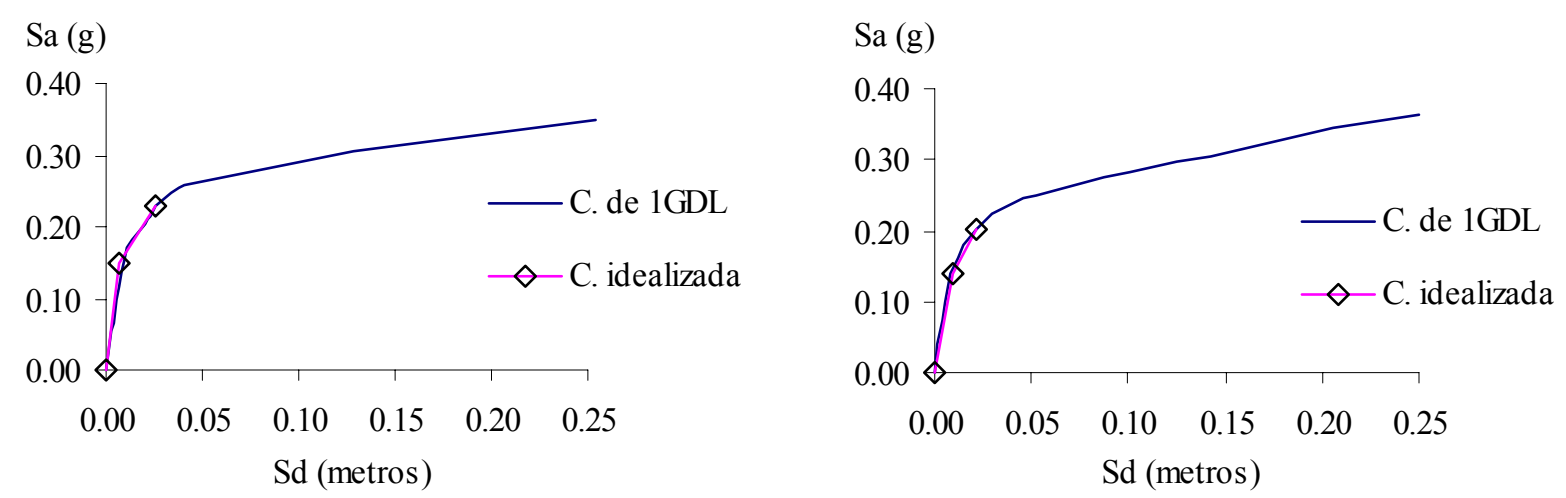

( c ) $\mathrm{e}_{\mathrm{s}}=0.2 \mathrm{~b}$

Figura 3. Curvas $\mathrm{S}_{\mathrm{a}}$ contra $\mathrm{S}_{\mathrm{d}} \mathrm{y}$ su idealización bilineal para el edificio de cuatro niveles. 


\section{Desplazamientos laterales máximos.}

En las siguientes figuras, al análisis propuesto basado en el análisis de empujón se le denomina "Empujón" y al análisis dinámico no-lineal paso a paso como "Dinámico". En la fig. 4 se muestran los desplazamientos laterales máximos en cada dirección del análisis para las tres excentricidades consideradas y se observa que, en general, los desplazamientos son muy similares con ambos análisis, excepto para la dirección X con excentricidad de $0.2 b$, en la que, los desplazamientos con el análisis de empujón son relativamente menores que los del análisis dinámico.
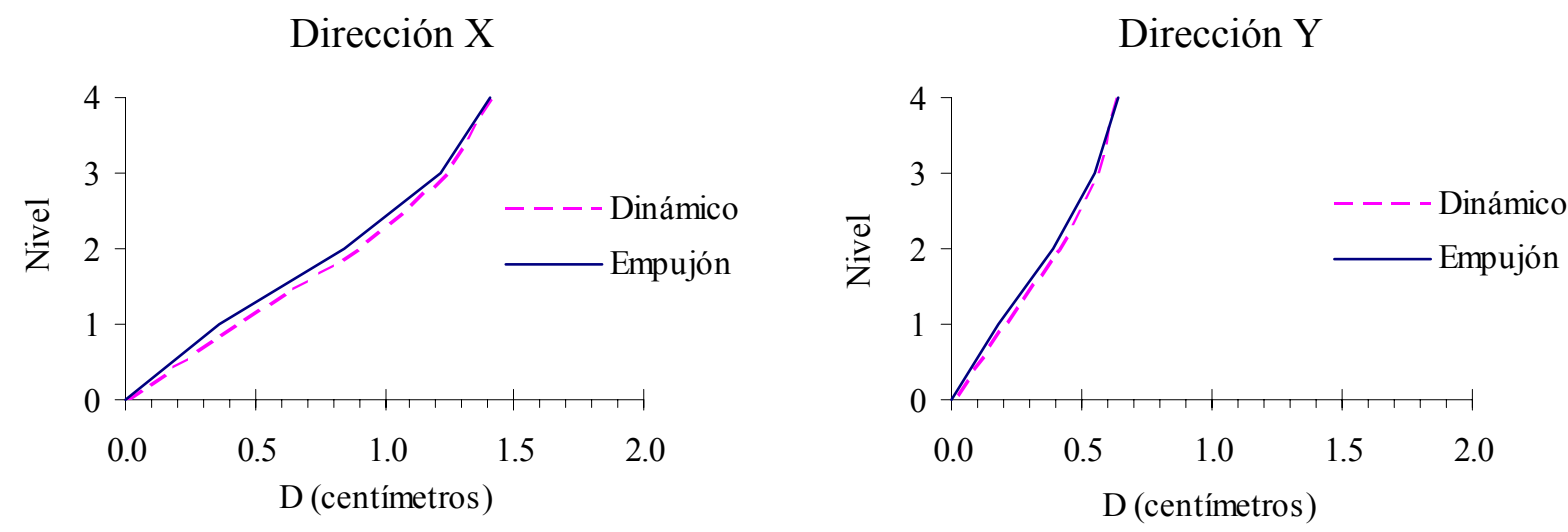

( a ) $\mathrm{e}_{\mathrm{s}}=(0.0 \mathrm{~b})$
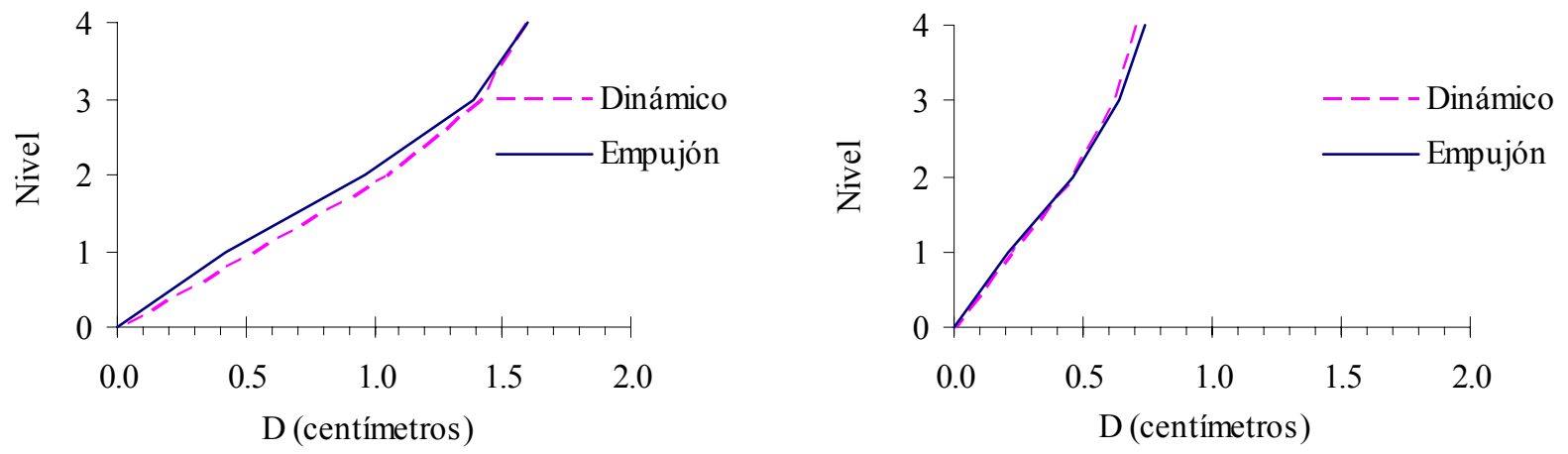

(b) $\mathrm{e}_{\mathrm{s}}=(0.1 \mathrm{~b})$
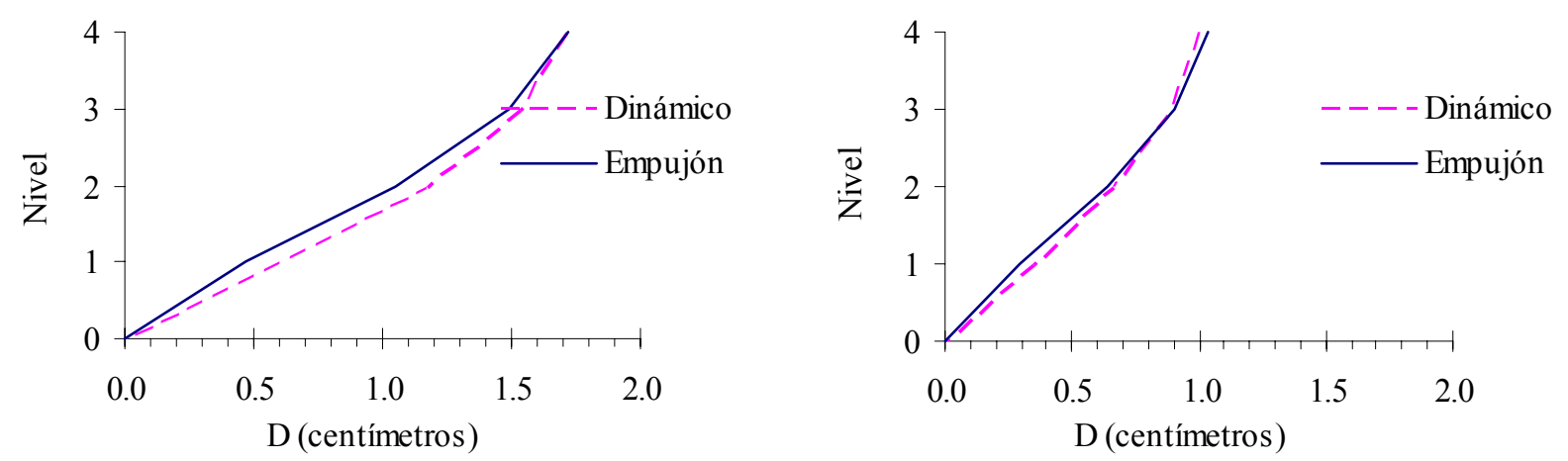

( c ) $\mathrm{e}_{\mathrm{s}}=(0.2 \mathrm{~b})$

Figura 4. Desplazamientos laterales máximos en los $\mathrm{CM}$ de los pisos. 
La relativa buena aproximación en los desplazamientos laterales no siempre ocurre en las rotaciones de los centros de masas de los pisos. La fig. 5 ilustra un caso extremo en el que la hipótesis de despreciar los momentos torsionantes en los pisos, producidos por la amplificación dinámica de la excentricidad, en los análisis de empujón conduce a diferencias significativas entre los resultados aproximados y los “exactos” provenientes de un análisis paso a paso.

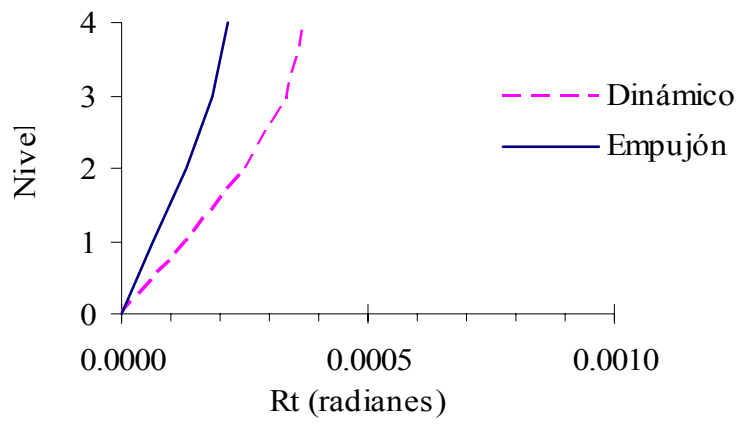

Figura 5. Rotaciones máximas en los $\mathrm{CM}$ de los pisos para $\mathrm{e}_{\mathrm{s}}=(0.1 \mathrm{~b})$.

Es de importancia mencionar que la amplificación dinámica de las excentricidades depende de las características dinámicas de la estructura por lo que para introducir en el análisis de empujón los momentos torsionantes en los pisos haría necesario el uso de resultados sobre la amplificación dinámica de las excentricidades estructurales en edificios asimétricos de varios niveles, para lo cual, desafortunadamente no existen resultados en la literatura especializada o bien realizar análisis modales de las estructuras completas lo que complicaría en exceso la aplicación de método resultante de evaluación.

\section{Distorsiones de entrepiso}

En la fig. 6 se muestran las distorsiones máximas de entrepiso en las direcciones X e Y para las tres excentricidades consideradas. En esta figura se observa que las distorsiones obtenidas con el método propuesto son mayores para los entrepisos 3 y 4, menores en el entrepiso 1 y muy similares con ambos análisis en el entrepiso 2.

\section{Articulaciones plásticas}

En las figs. 7 y 8 se ilustran, resultado del análisis de empujón y dinámico no-lineal para tres casos considerados, la localización de las articulaciones plásticas así como las magnitudes de las ductilidades de rotación en los extremos de trabes y columnas de los marcos A y 1 de la fig. 1, respectivamente. En la tabla 1 se muestra la representación gráfica de las magnitudes de las ductilidades de articulación plástica.

Al comparar los resultados de la fig. 7 se observa que las distribuciones de articulaciones plásticas con el análisis de empujón son muy similares a las obtenidas con el análisis dinámico. Con respecto a las magnitudes de las articulaciones plásticas, se observa que las obtenidas con el 
análisis dinámico son ligeramente mayores en las trabes del primer nivel y en las columnas en la base del primer entrepiso, las trabes del segundo nivel son similares entre ambos análisis y en el tercer nivel la magnitud de ductilidades en trabes son ligeramente mayores con el análisis de empujón.
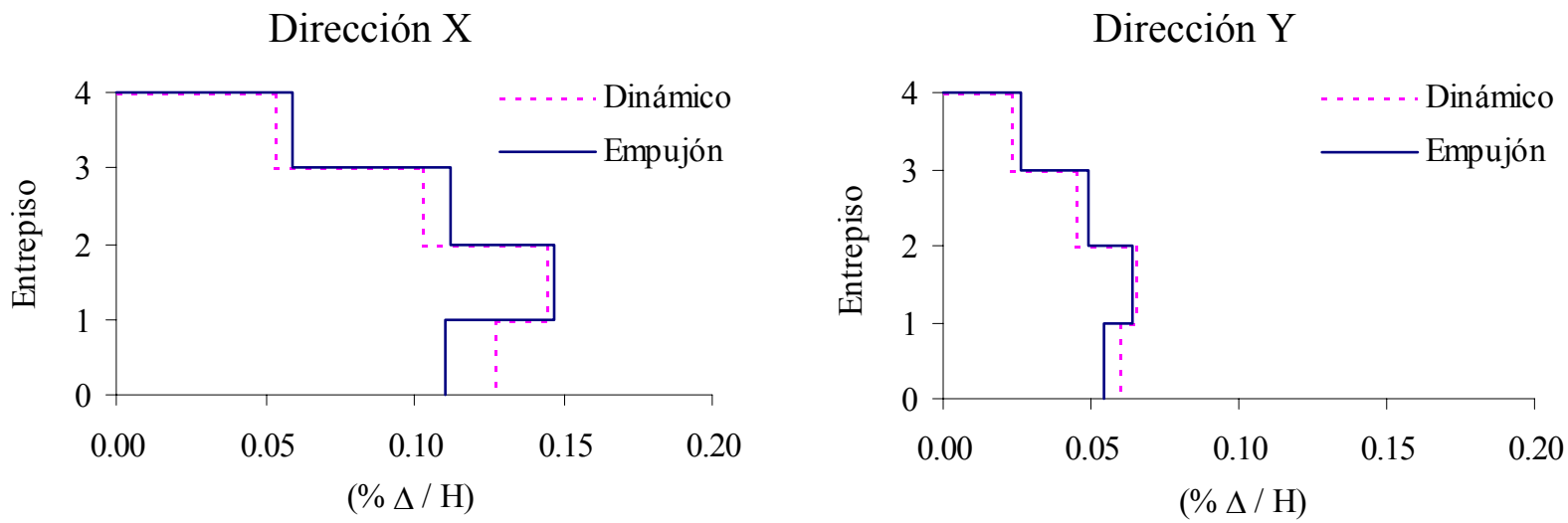

( a ) $\mathrm{e}_{\mathrm{s}}=(0.0 \mathrm{~b})$
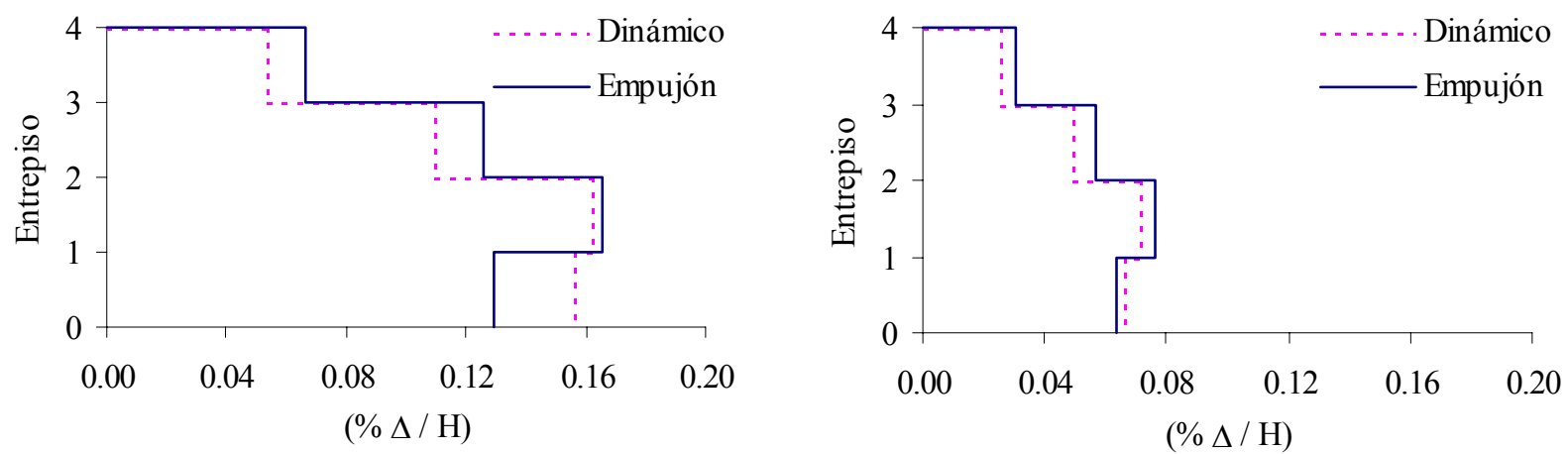

( b ) $e_{s}=(0.1 b)$
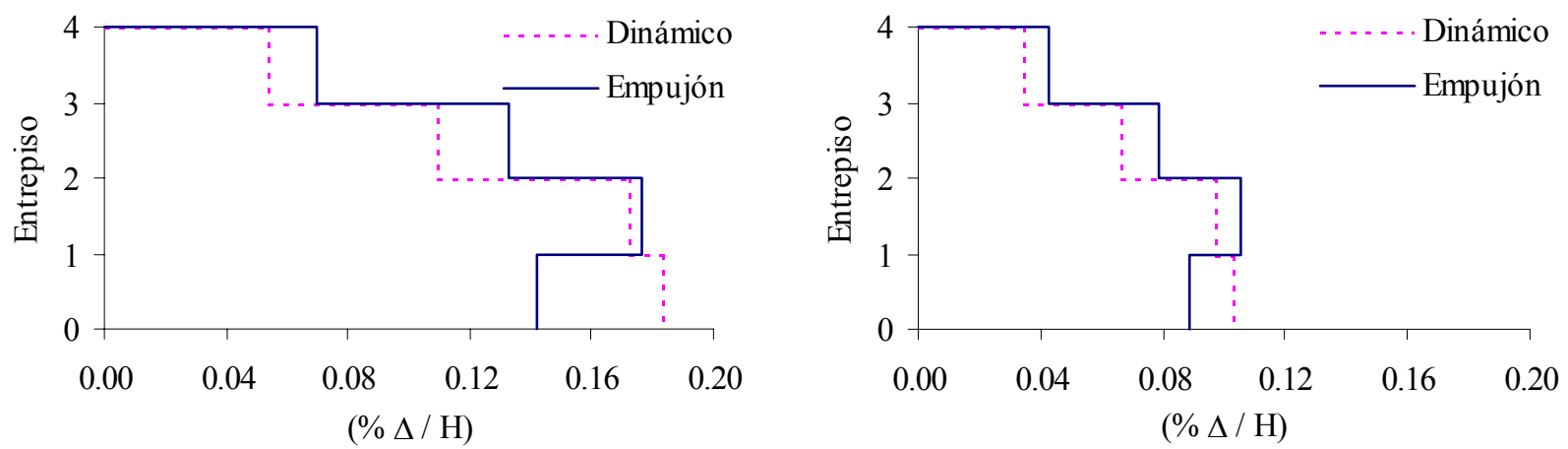

\section{( c ) $\mathrm{e}_{\mathrm{s}}=(0.2 \mathrm{~b})$}

Figura 6. Distorsiones de entrepiso en los $\mathrm{CM}$ de los pisos.

En la fig. 8 se observa que aparecen un mayor número de articulaciones plásticas con el análisis dinámico, ya que para el modelo con excentricidad $e_{s}=0.0 \mathrm{~b}$ se articulan dos trabes mientras que con el análisis de empujón solo una; en el modelo con excentricidad $\mathrm{e}_{\mathrm{s}}=0.1 \mathrm{~b}$, con el 
análisis dinámico se articulan todas las columnas en la base del primer entrepiso y con el análisis de empujón ninguna; y en el modelo con excentricidad $\mathrm{e}_{\mathrm{s}}=0.2 \mathrm{~b}$ con el análisis dinámico se presentan en la parte superior de las columnas centrales del segundo entrepiso dos articulaciones más que en el análisis de empujón. Las magnitudes de las ductilidades en articulaciones plásticas obtenidas con el análisis dinámico son muy similares a las obtenidas con el análisis de empujón para aquellos elementos donde se presentan.

Tabla 1. Magnitudes de las ductilidades de rotación plástica.

\begin{tabular}{|c|c|c|c|c|c|}
\hline 1 & 2 & 3 & 4 & 5 & 6 \\
\hline$\bullet$ & $\bullet$ & - & - & 0 & 0 \\
\hline
\end{tabular}

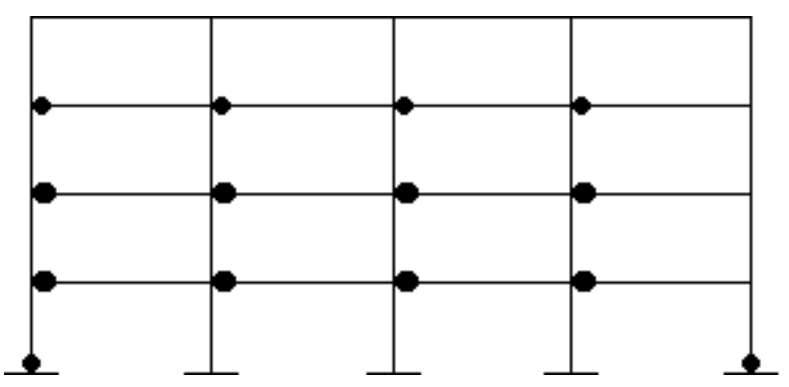

Análisis de empujón.

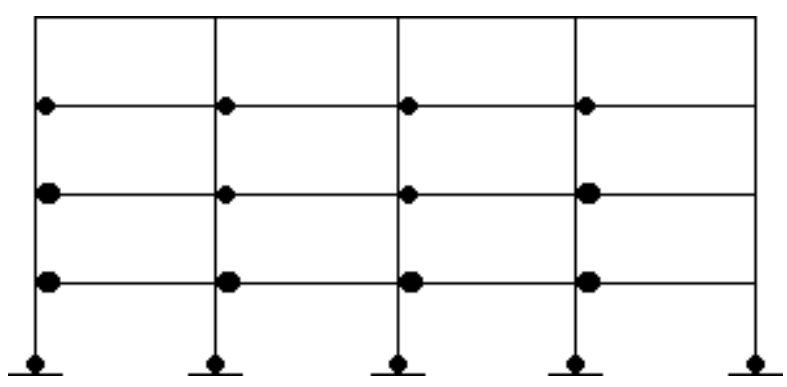

Análisis dinámico.

\section{( a ) $\mathrm{e}_{\mathrm{s}}=0.0 \mathrm{~b}$}

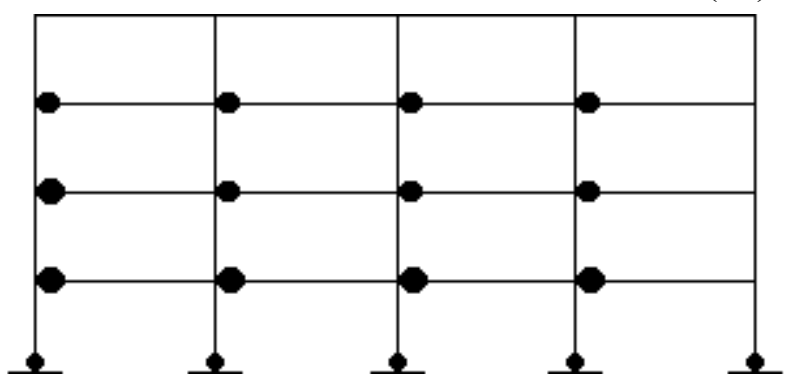

Análisis de empujón.

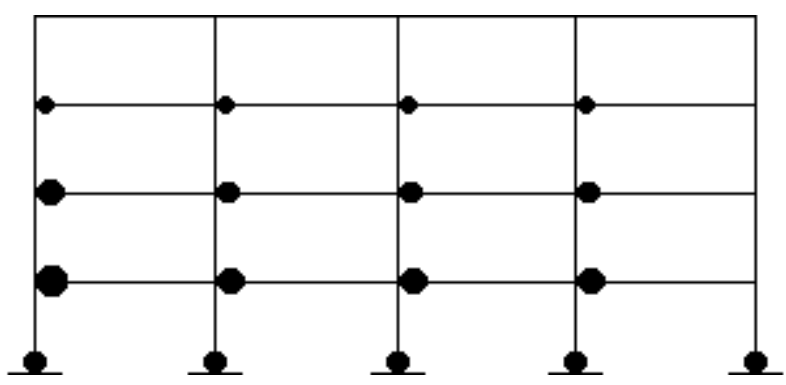

Análisis dinámico.

( b ) $\mathrm{e}_{\mathrm{s}}=0.1 \mathrm{~b}$

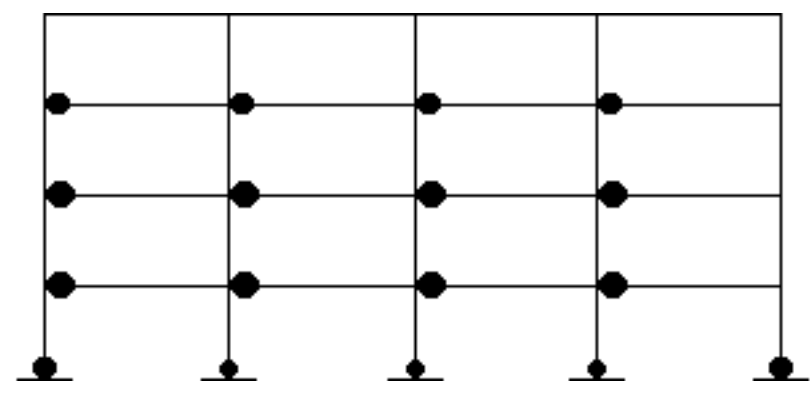

Análisis de empujón.

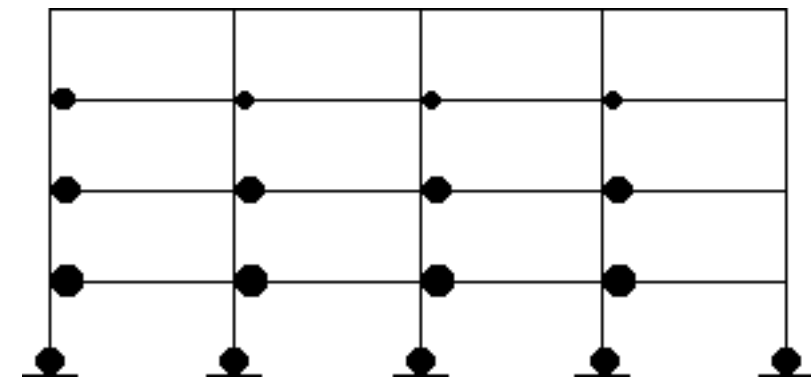

Análisis dinámico.

(c ) $\mathrm{e}_{\mathrm{s}}=0.2 \mathrm{~b}$

Figura 7. Distribución de articulaciones para el marco A. 


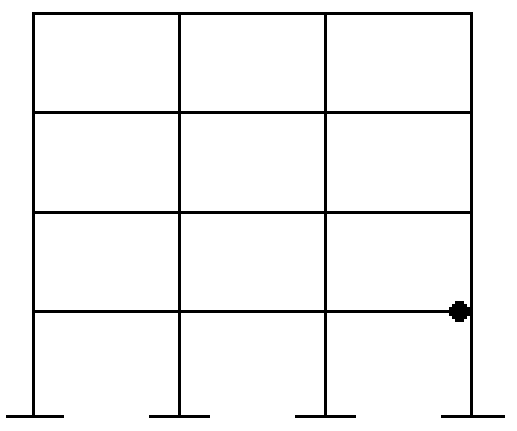

Análisis de empujón.

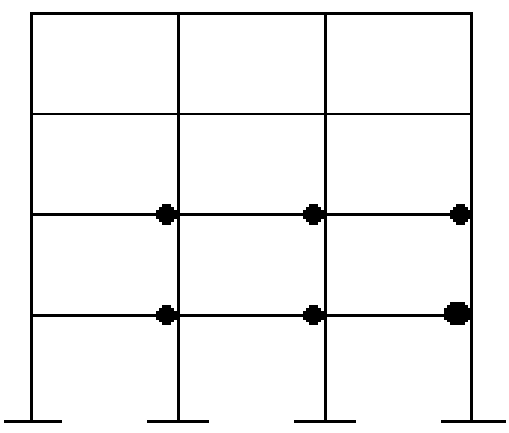

Análisis de empujón.

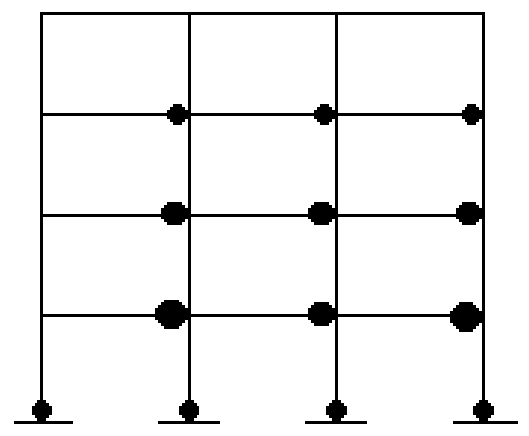

Análisis de empujón.

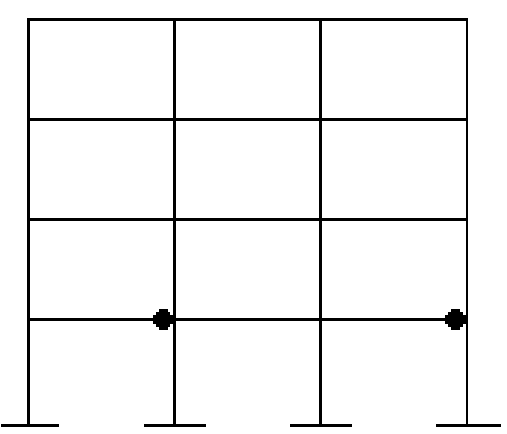

Análisis dinámico.

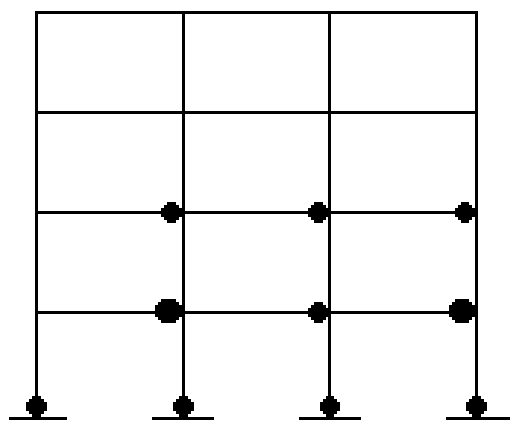

Análisis dinámico.

( b ) $\mathrm{e}_{\mathrm{s}}=0.1 \mathrm{~b}$

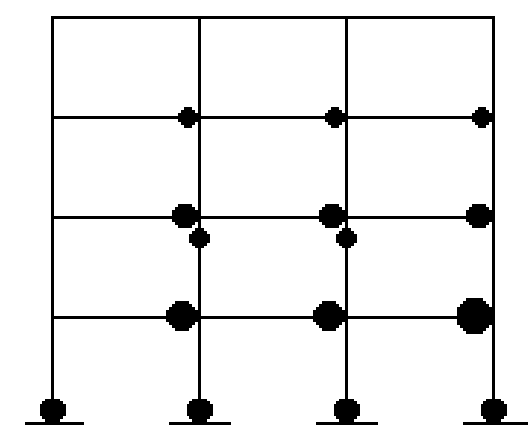

Análisis dinámico.

( c ) $\mathrm{e}_{\mathrm{s}}=0.2 \mathrm{~b}$

Figura 8. Distribución de articulaciones para el marco 1.

\section{EDIFICIO DE OCHO NIVELES}

Procediendo de la misma manera que para el edificio de cuatro niveles, en la fig. 9 se muestran las curvas de capacidad para las tres excentricidades consideradas en el edificio de ocho niveles. Se observa que por los mismos motivos que en el edificio de cuatro niveles en la dirección de evaluación $\mathrm{X}$ las curvas de capacidad son muy similares para las diferentes excentricidades. En la dirección de evaluación Y se observa que al aumentar la excentricidad de las estructuras, la rigidez y la resistencia del edificio disminuyen. 


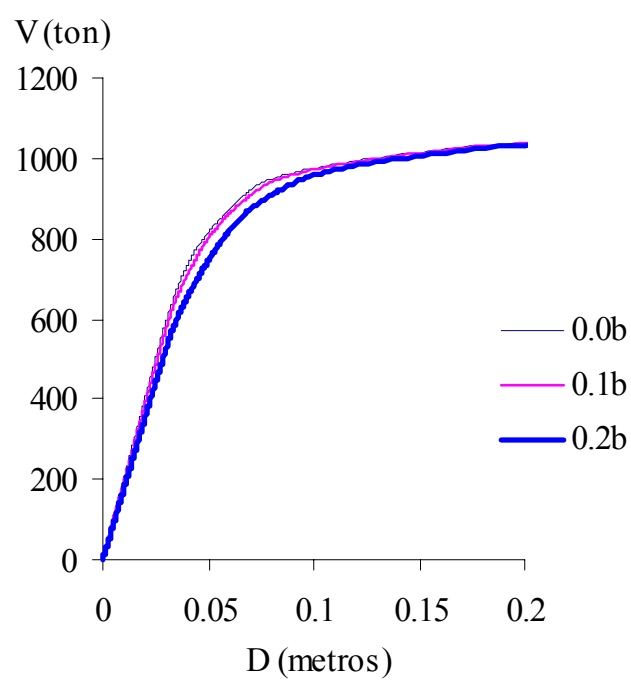

(a) Dirección X.

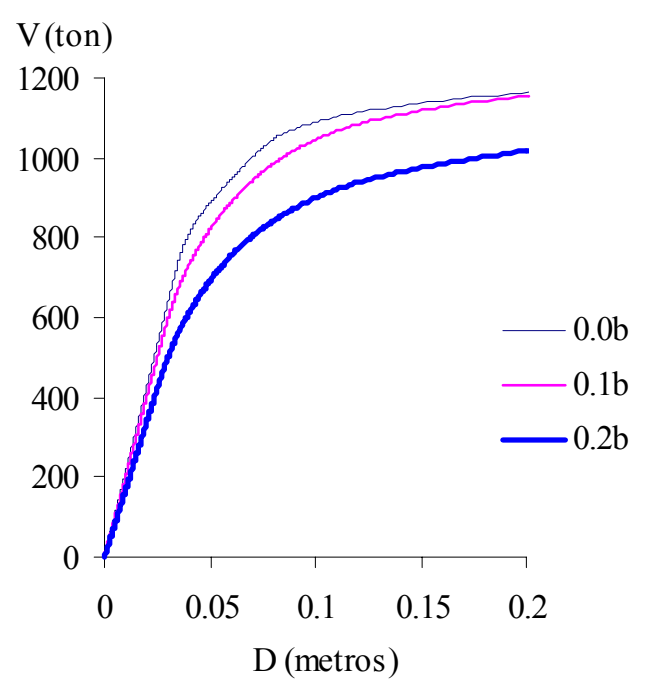

( b ) Dirección Y.

Figura 9. Curvas de capacidad para el edificio de ocho niveles.

En la fig. 10 se ilustran las curvas $S_{a}$ contra $S_{d}$ de los sistemas de 1GDL para los casos considerados y sus idealizaciones bilineales correspondientes a los desplazamientos espectrales máximos que definen los puntos de comportamiento.

En la obtención de cualquiera de los puntos de comportamiento de las curvas de la fig. 10 se supone uno y con la curva bilineal resultante se obtiene uno nuevo punto, si al revisar su valor con el originalmente propuesto este no coincide, este procedimiento se repite hasta que el punto de comportamiento resulte suficientemente cercano al supuesto. Una vez determinado el desplazamiento espectral máximo para cada dirección de evaluación (punto de comportamiento), se calcula el correspondiente desplazamiento de azotea y con él se efectúan el análisis de empujón.

\section{Desplazamientos laterales máximos.}

En la fig. 11 se muestran los resultados obtenidos de los análisis de empujón para los desplazamientos laterales máximos en cada dirección de análisis para las tres excentricidades consideradas. Al compararlos con los resultados de un análisis no lineal paso a paso se observa que los desplazamientos del análisis paso a paso son mayores a los obtenidos del análisis de empujón, excepto en los niveles 7 y 8 del modelo con excentricidad $\mathrm{e}_{\mathrm{s}}=0.1 \mathrm{~b}$ y dirección de evaluación X. Se observa también que para el modelo $e_{\mathrm{s}}=0.0 \mathrm{~b}$ en la dirección de evaluación $\mathrm{Y}$ los desplazamientos con ambos análisis son muy similares.

Las diferencias en los desplazamientos laterales para ambos métodos pueden deberse a que en el método aproximado se considera que la estructura vibra lateralmente en sus modos fundamentales desacoplados y por lo tanto se empujó con la distribución correspondiente a estos 
modos, despreciando la contribución que los modos superiores tienen en las cargas de los pisos inferiores.

\section{Dirección X}

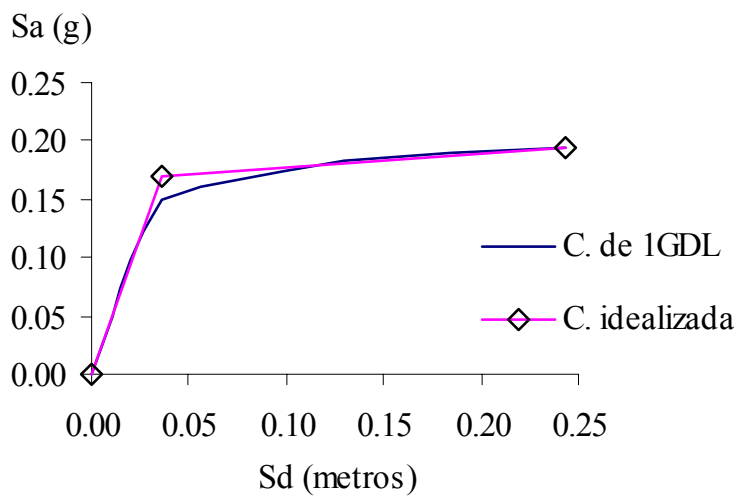

\section{Dirección Y}

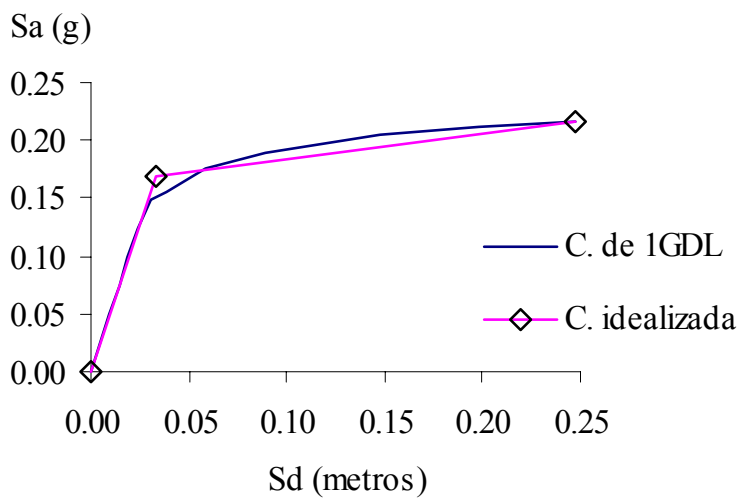

( a ) $\mathrm{e}_{\mathrm{s}}=0.0 \mathrm{~b}$
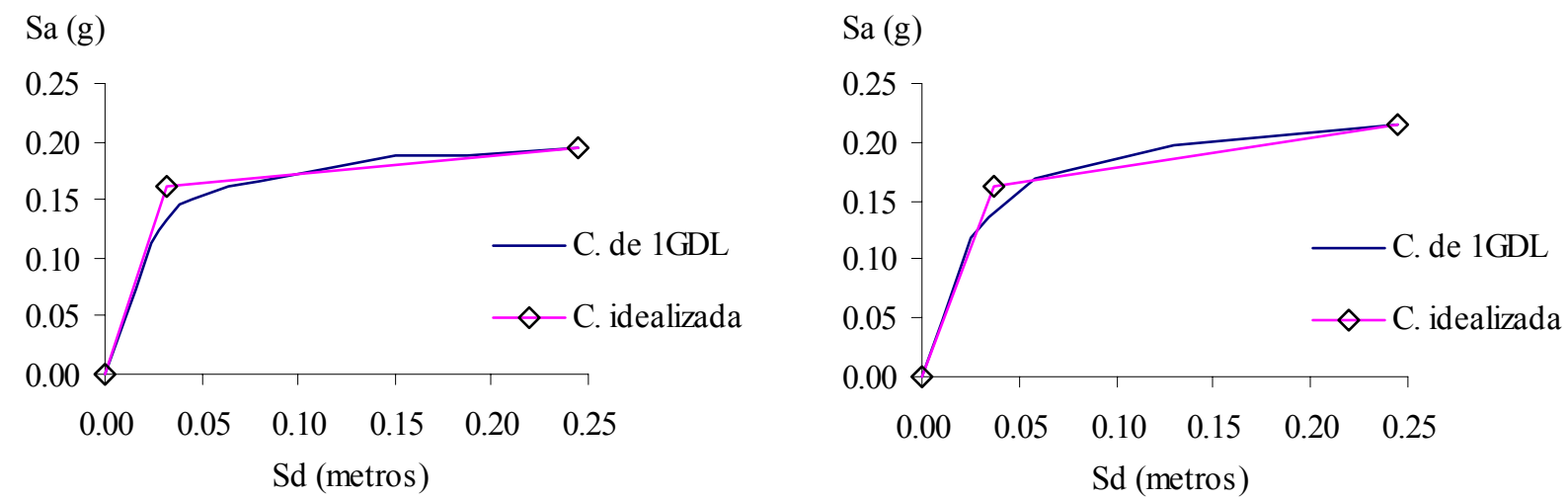

( b ) $\mathrm{e}_{\mathrm{s}}=0.1 \mathrm{~b}$
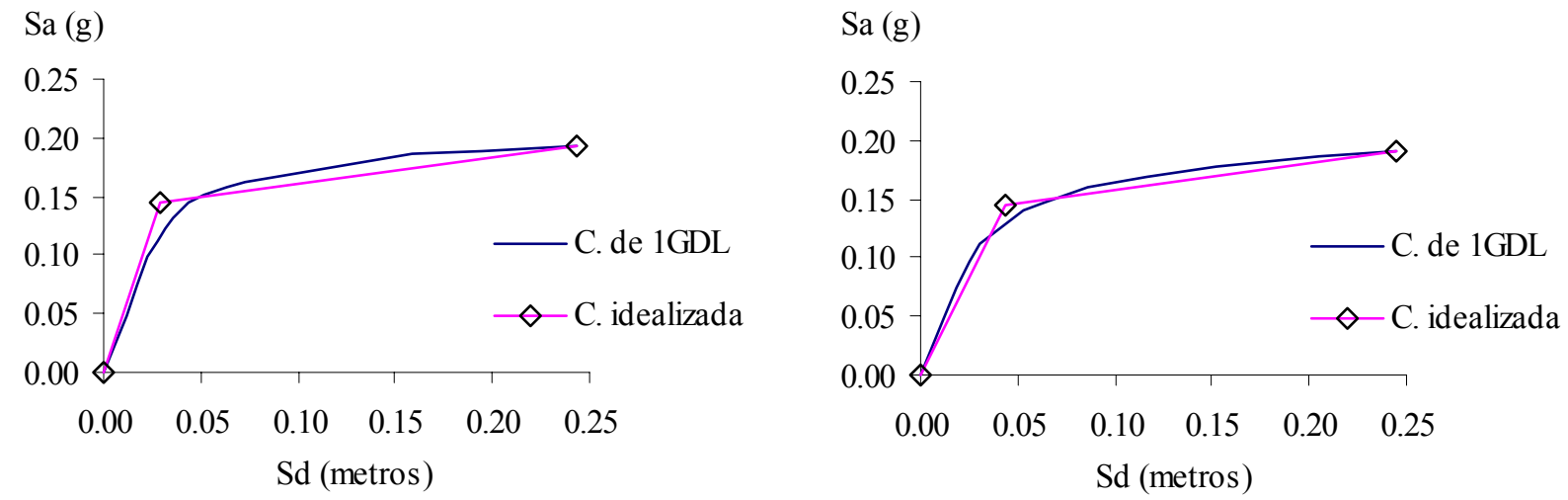

( c ) $e_{s}=0.2 b$

Figura 10. Curvas $S_{a}$ contra $S_{d}$ y sus idealizaciones bilineales para el edificio de ocho niveles.

En relación con las rotaciones de los pisos en la fig. 2 se presentan los resultados obtenidos para una excentricidad estructural de los pisos de $0.1 \mathrm{~b}$. Contrariamente a lo que se 
mostró en la fig. 5, en este caso la aproximación obtenida en las rotaciones de los pisos es aceptable. Los resultados mostrados de esta figura permiten suponer que en este caso el factor de amplificación dinámico de las excentricidades seguramente es cercano a 1 .

\section{Dirección X}

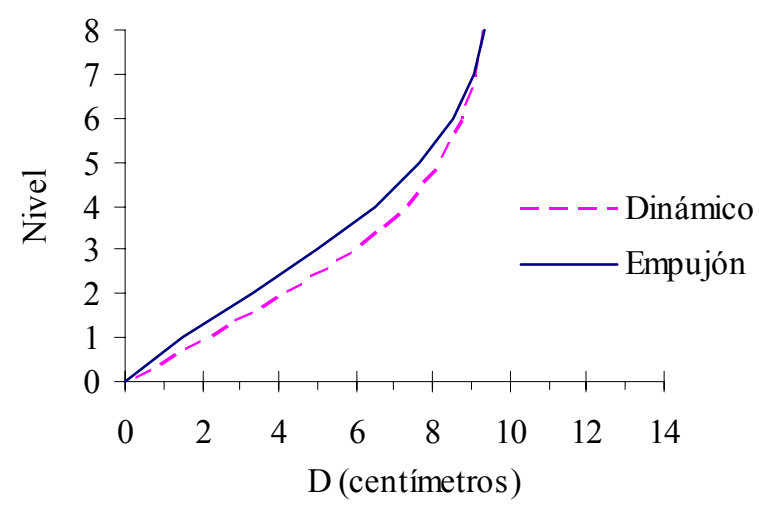

\section{Dirección Y}

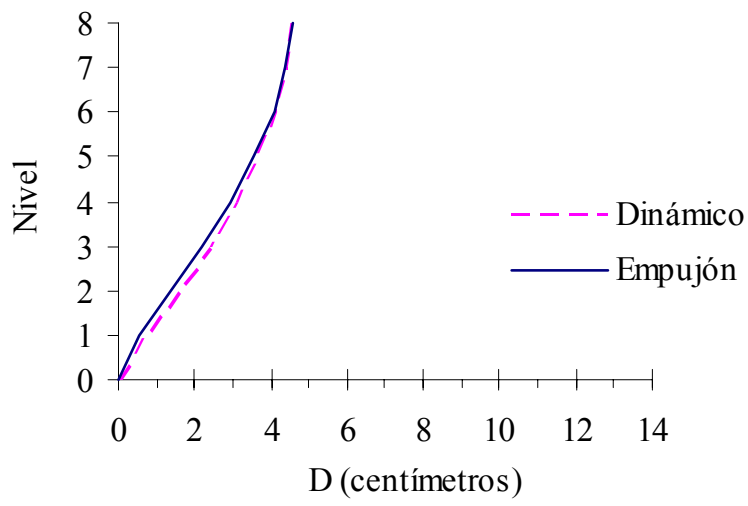

( a ) $\mathrm{e}_{\mathrm{s}}=(0.0 \mathrm{~b})$

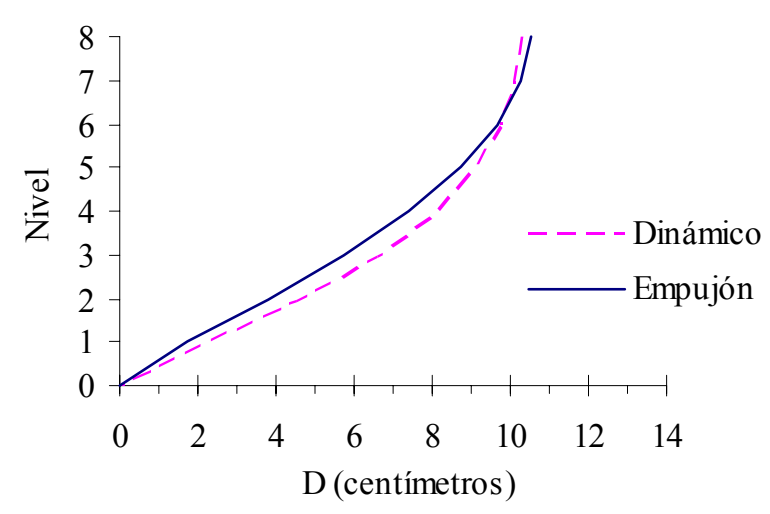

( b ) $\mathrm{e}_{\mathrm{s}}=(0.1 \mathrm{~b})$

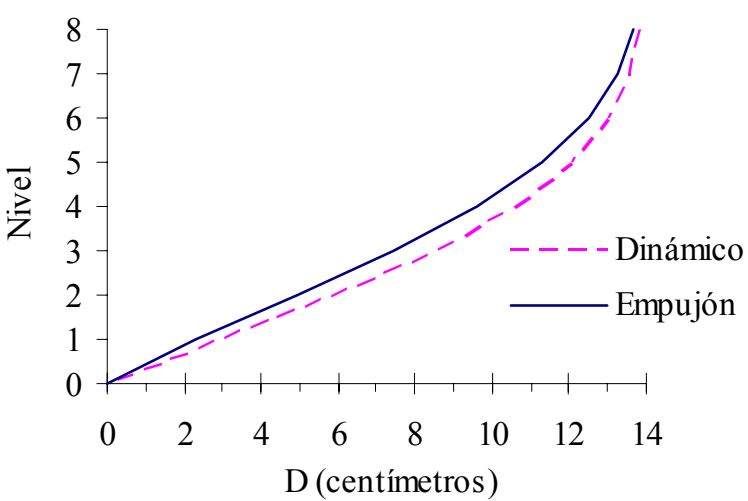

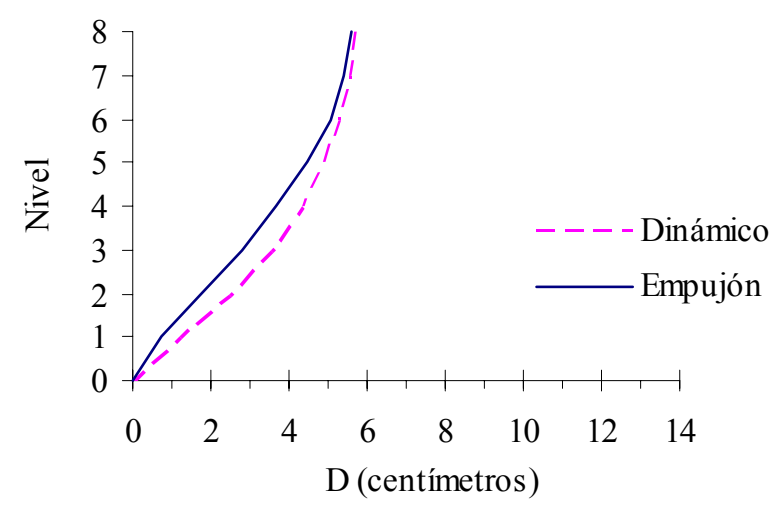

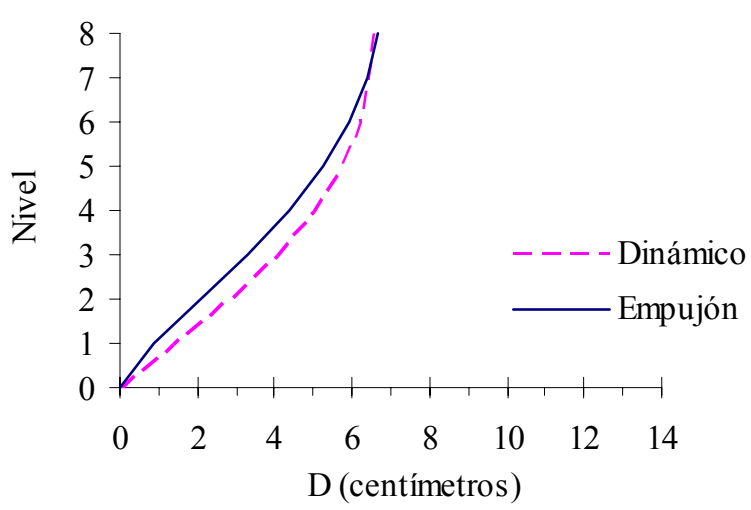

( c ) $\mathrm{e}_{\mathrm{s}}=(0.2 \mathrm{~b})$

Figura 11. Desplazamientos laterales máximos en los $\mathrm{CM}$ de los pisos. 


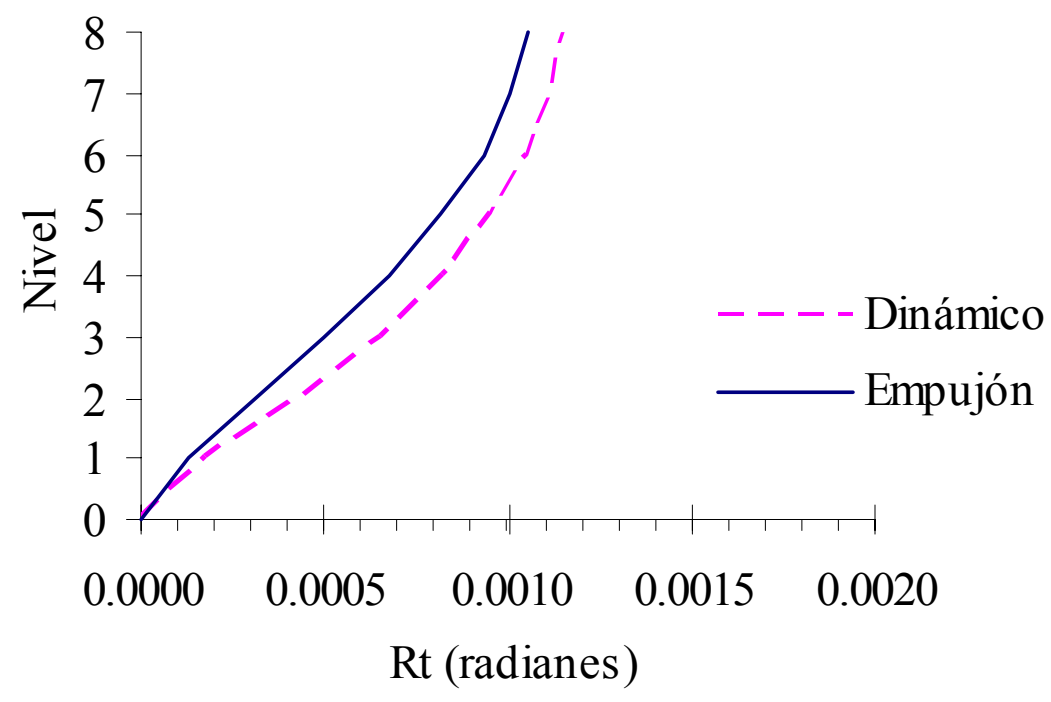

Figura 12. Rotaciones máximas en los $\mathrm{CM}$ de los pisos para $\mathrm{e}_{\mathrm{s}}=(0.1 \mathrm{~b})$

\section{Distorsiones de entrepiso}

En la fig. 13 se muestran las distorsiones máximas de entrepiso en las direcciones X e Y para las tres excentricidades consideradas. En esta figura se observa que las distorsiones obtenidas con el método propuesto son mayores excepto en los entrepisos 1, 2 y 3 donde las distorsiones del análisis dinámico son mayores.

\section{Articulaciones plásticas}

En las figs. 14 y 15 se ilustran para los tres casos considerados las distribuciones de articulaciones plásticas y las magnitudes de sus ductilidades de rotación plástica para los marcos A y 1 de la fig. 1, respectivamente.

De la información presentada en las figs. 14 y 15 se puede concluir que, aunque no idénticas, la distribución y magnitudes de las articulaciones en trabes y columnas son en general similares para ambos métodos. Se observa, sin embargo, que con el método aproximado se articularon más las trabes que con el método paso a paso y que en columnas sucedió lo contrario.

Al igual que para el edificio de cuatro niveles, en el análisis del empujón del de ocho niveles se buscó que la orientación de fuerzas en los centros de masa y en cada dirección provocara las condiciones más desfavorables a cada marco. Es importante puntualizar, que desde el punto de vista de evaluación del comportamiento estructural, los resultados del método aproximado representan valores extremos que cubren todos los posibles escenarios bajo las cuales se debe evaluar la estructura. 
Dirección X

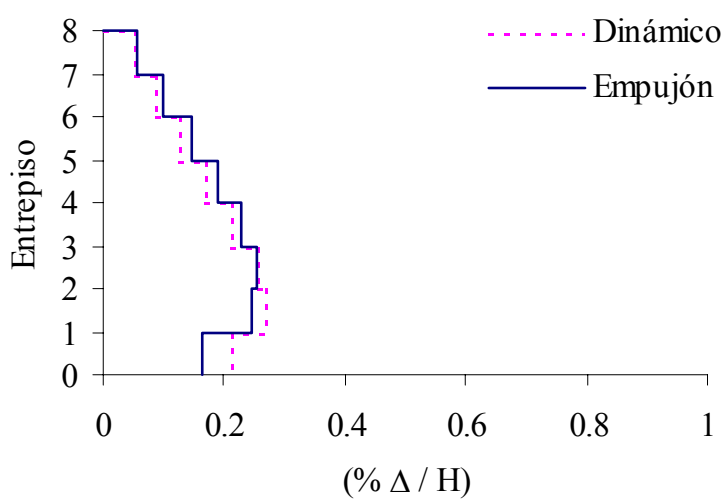

( a ) $\mathrm{e}_{\mathrm{s}}=(0.0 \mathrm{~b})$

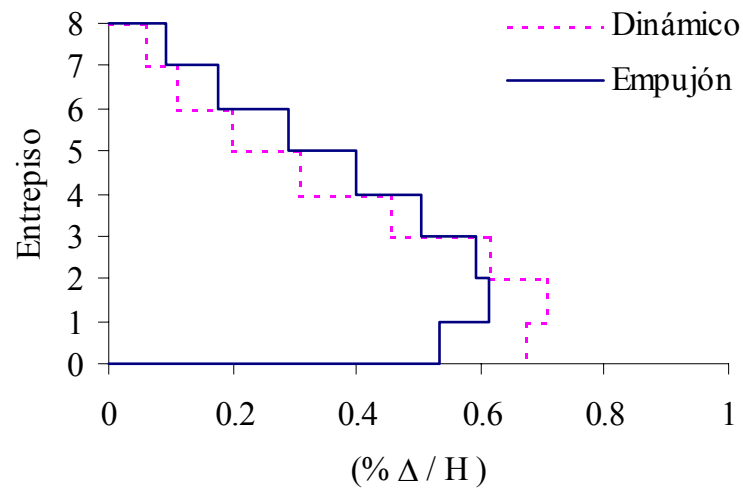

( b ) $e_{s}=(0.1 b)$
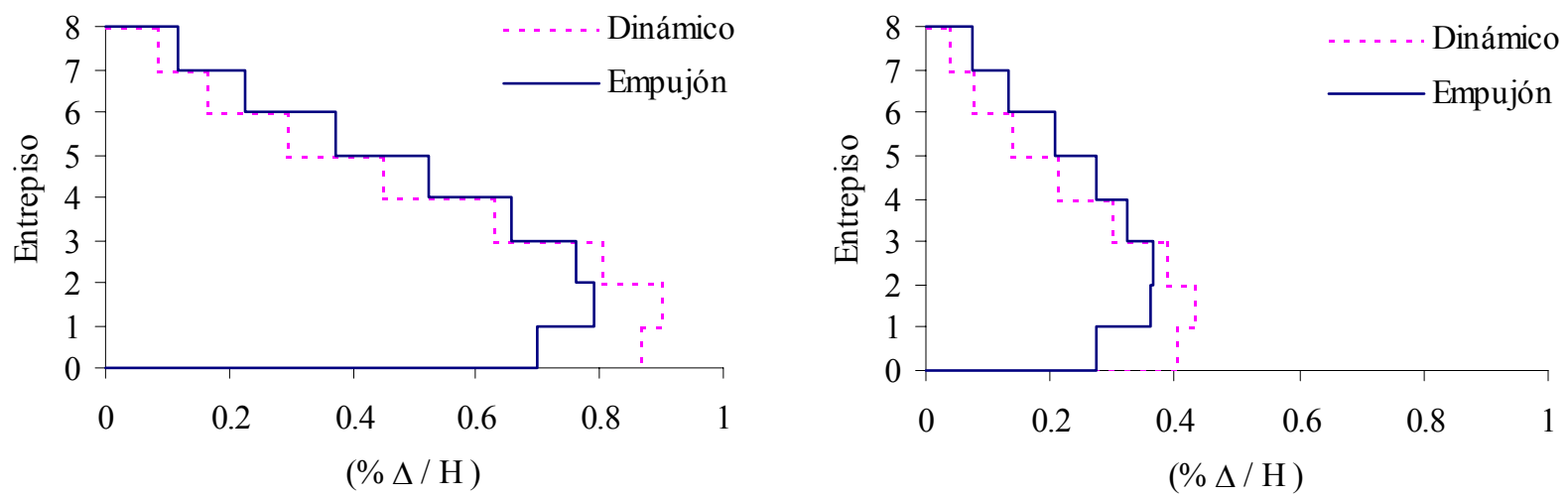

( c ) $\mathrm{e}_{\mathrm{s}}=(0.2 \mathrm{~b})$

Figura 13. Distorsiones de entrepiso en los CM de los pisos. 


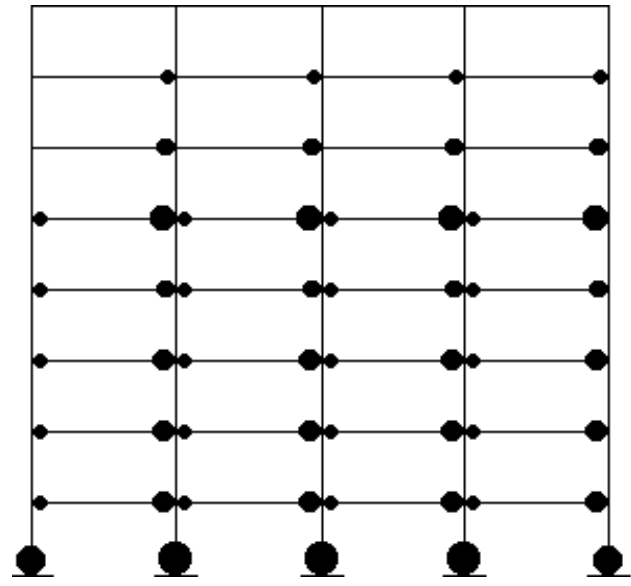

Análisis de empujón.

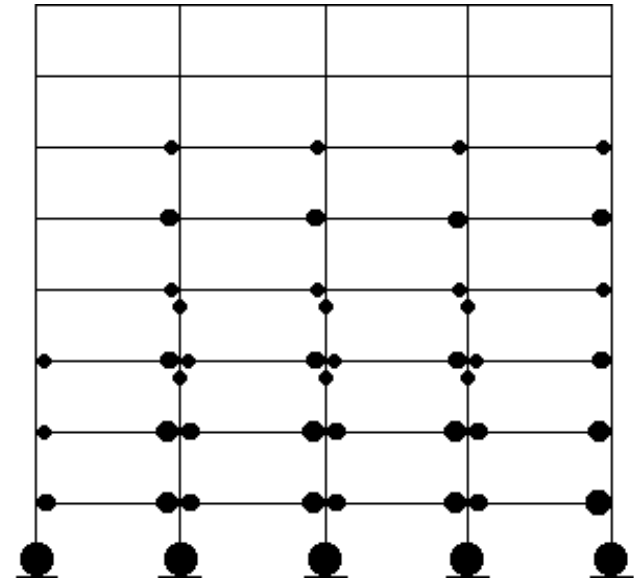

Análisis dinámico

( a ) $\mathrm{e}_{\mathrm{s}}=0.0 \mathrm{~b}$

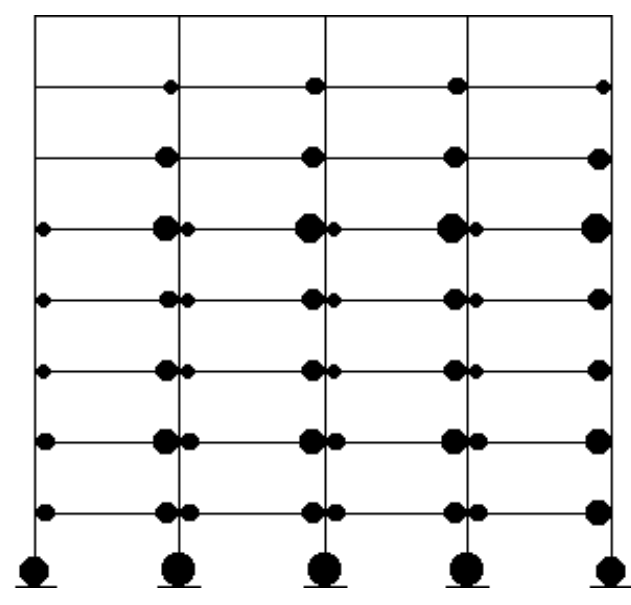

Análisis de empujón.

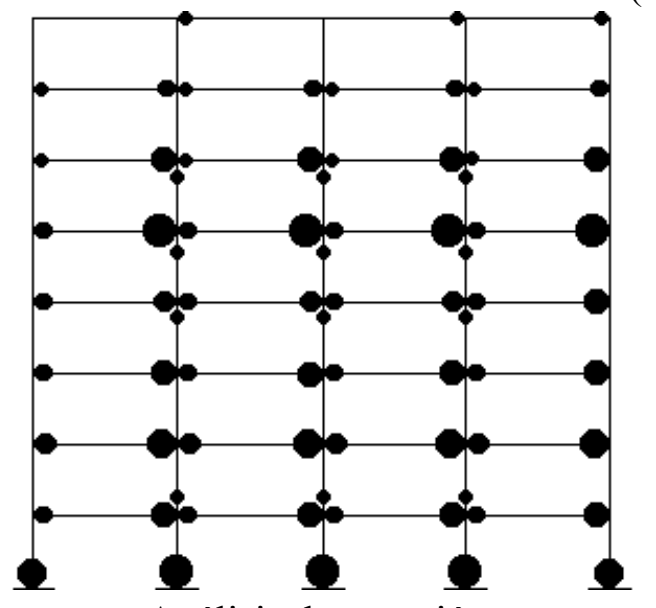

Análisis de empujón.

(b) $e_{s}=0.1 b$

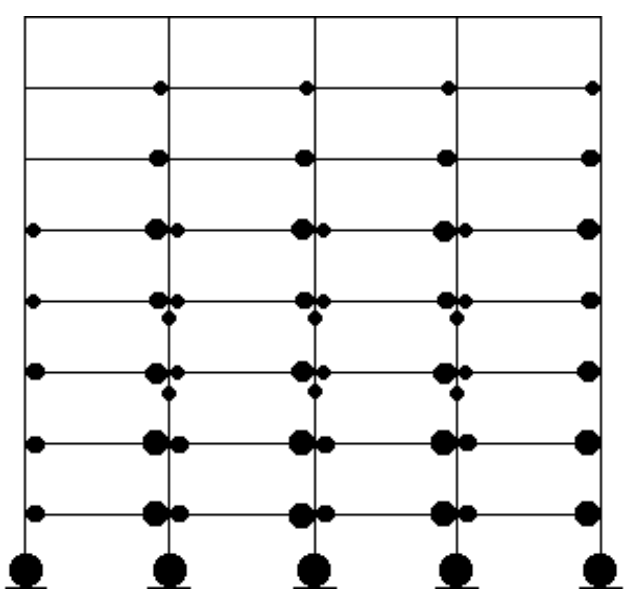

Análisis dinámico (c ) $e_{s}=0.2 b$

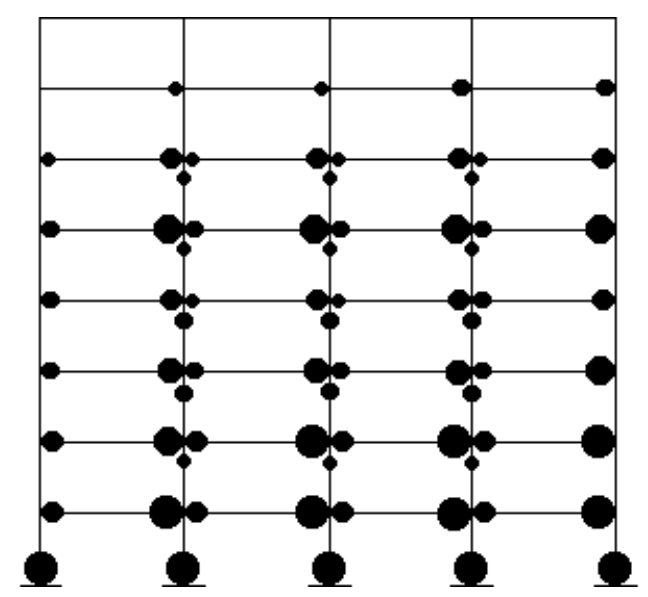

Análisis dinámico

Figura 14. Distribución de articulaciones en el marco A. 


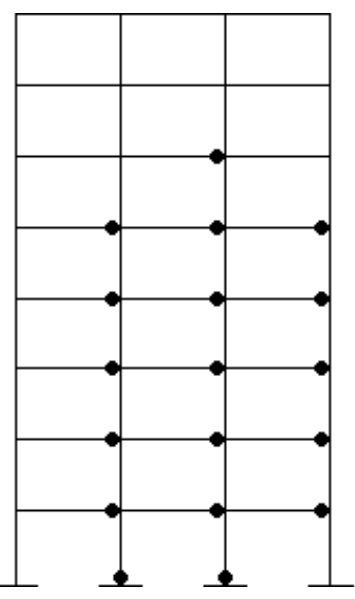

Análisis de empujón.

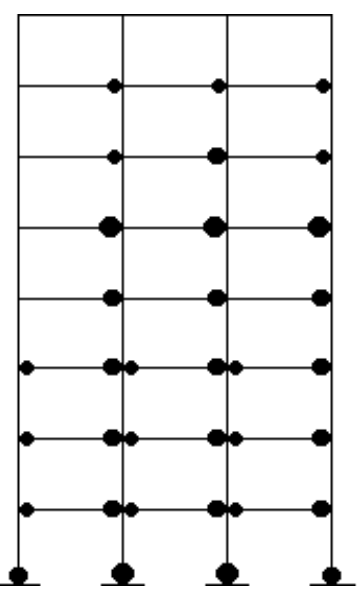

Análisis de empujón.

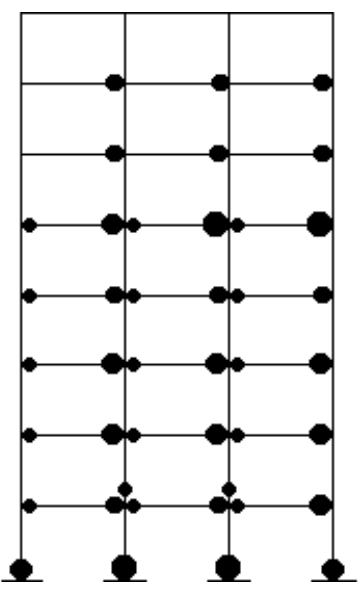

Análisis de empujón.

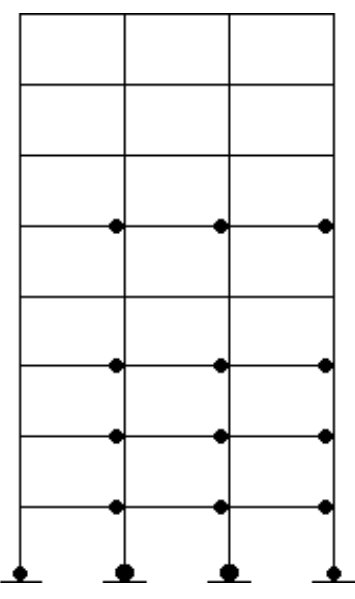

Análisis dinámico

( a ) $\mathrm{e}_{\mathrm{s}}=0.0 \mathrm{~b}$

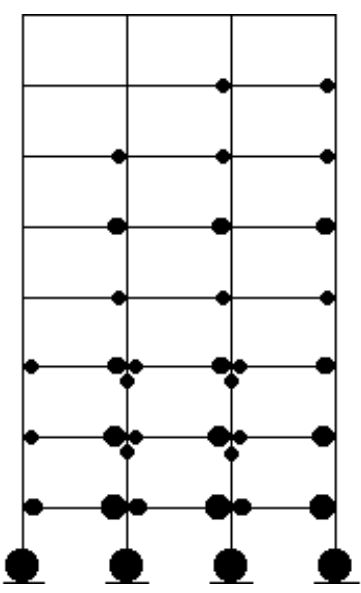

Análisis dinámico

( b ) $\mathrm{e}_{\mathrm{s}}=0.1 \mathrm{~b}$

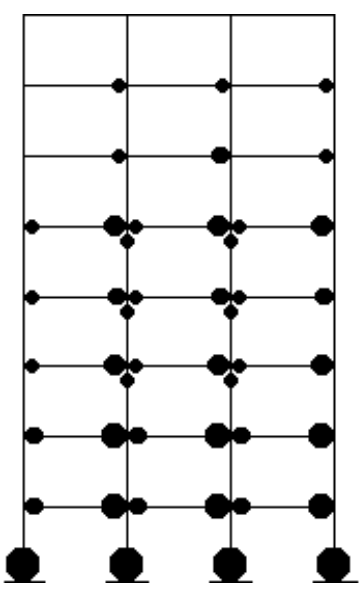

Análisis dinámico

(c ) $\mathrm{e}_{\mathrm{s}}=0.2 \mathrm{~b}$

Figura 15. Distribución de articulaciones en el marco 1. 


\section{CONCLUSIONES Y RECOMENDACIONES}

En este trabajo se presenta un nuevo procedimiento simplificado de evaluación del desempeño sísmico no-lineal de estructuras en 3D de edificios asimétricos. Para ilustrar la aplicación de este procedimiento como una herramienta de evaluación se estudian ejemplos de edificios asimétricos en los que la asimetría se proporciona desplazando los centros de masas de los pisos. Los ejemplos considerados correspondieron a edificios de cuatro y ocho niveles previamente diseñados de acuerdo a un código vigente. Del trabajo realizado se puede concluir lo siguiente:

Un resultado importante del análisis del empujón, requerido por el método, es que la curva de capacidad, propiedad estructural, proporciona información acerca de la rigidez inicial, de la rigidez de post-fluencia, de la resistencia de la estructura y de la evolución del daño hasta el colapso para niveles crecientes de cortante basal.

En todos los casos estudiados, el método propuesto predijo con excelente aproximación el desplazamiento máximo de los niveles superiores, no siendo así para los niveles inferiores donde los desplazamientos obtenidos del análisis dinámico fueron mayores que los del método propuesto. Lo anterior, en principio, se atribuye a los alcances de la hipótesis de trabajo de este estudio la cual lleva a resultados aproximados aceptables sólo para niveles de excentricidad reducidos.

Es importante puntualizar que los ejemplos seleccionados para ilustrar el método fueron diseñados como estructuras simétricas, lo cual justifica la aparición de articulaciones plásticas en la base de las columnas, lo que en principio contradice el criterio de columna fuerte - viga débil, base de la filosofía actual de diseño sísmico. Sin embargo, se considera que la abundancia de articulaciones y magnitud de las rotaciones plásticas obtenidas representan una prueba satisfactoria del potencial del método aproximado para predecir el desempeño sísmico de edificios asimétricos.

En lo referente a la formación de articulaciones plásticas en vigas y columnas, el método aproximado predice su localización, solo que las magnitudes de las ductilidades son menores que las correspondientes de un análisis dinámico paso a paso.

Al comparar los resultados correspondientes a una estructura sometida a sismos de diferentes intensidades o bien a estructuras diferentes que experimentan efectos inelásticos diferentes sometidas a un mismo sismo con los "exactos" se encuentra que su calidad se deteriora con efectos inelásticos crecientes.

El procedimiento propuesto de análisis simplificado en 3D, basado en reducir un modelo de VGDL en un sistema equivalente de 1GDL correspondiente al modo fundamental de la estructura, puede ser eficazmente usado para predecir la respuesta de una estructura por indicadores de respuesta global dados por los desplazamientos máximos de los niveles y las distorsiones de entrepiso. Las demandas máximas de ductilidad en vigas y columnas son indicadores del daño estructural y representan la respuesta local de los elementos. 
Del análisis de los resultados presentados en este trabajo se derivan las siguientes recomendaciones:

Es necesario llevar a cabo más estudios sobre estructuras asimétricas en las que la excentricidad sea debida a las distribuciones en planta de las rigideces. De igual manera es de interés evaluar el método para estructuras con excentricidad en resistencias.

Es necesario realizar estudios refinados sobre la regla de combinación de acciones bidireccionales, ya que ni la que especifica el RCDF-93 (DDF, 1993), empleada en este estudio, ni las otras recomendadazas por otros códigos, necesariamente representan las proporciones observadas en registros en el Valle de México. La importancia que tiene esta regla en la obtención de la curva de capacidad puede en principio ser la causa más importante en las diferencias observadas entre los resultados aproximados y los "exactos".

Para obtener resultados más refinados con el método propuesto, particularmente para niveles elevados de excentricidad, se recomienda empujar la estructura con una distribución de cargas horizontales en la cual se incluyan los efectos de los modos superiores. Esta distribución se puede determinar combinando los modos o las cargas mediante la regla de superposición modal de la raíz cuadrada de la suma de los cuadrados, SRSS o la combinación cuadrática completa, CQC.

Otro aspecto importante a considerar en investigaciones futuras es evaluar con mayor detalle el efecto de los cambios en la distribución de la carga horizontal debidos a los cambios en el modo fundamental, ocasionados por la aparición de daño durante el proceso del análisis de empujón.

\section{RECONOCIMIENTOS}

Se agradece al Consejo Nacional de Ciencia y Tecnología las becas del segundo y tercer autores y el patrocinio de este trabajo, parte del proyecto "Desarrollo de métodos analíticos y experimentales para la evaluación del comportamiento sísmico de estructuras". De igual manera se agradece a la Dirección General de Asuntos Académicos de la UNAM el patrocinio del proyecto "Desarrollo de criterios de diseño sísmico por torsión" y la beca del segundo autor.

\section{REFERENCIAS}

ATC (1996), "Seismic evaluation and retrofit of concrete buildings", Reporte ATC-40, Applied Technology Council, Redwood City, California, Estados Unidos.

Ayala, M (1999), "Evaluación del comportamiento sísmico de estructuras de edificios asimétricos en 3D”, Tesis de Maestría, DEPFI, UNAM, México.

Charney, F (1998), "NONLIN. Nonlinear dynamic time history analysis of single degree of freedom systems", Advanced Structural Concepts, Inc., Golden, Colorado, Estados Unidos. 
Chopra, A K (2001), Dynamics of structures. Theory and applications to earthquake engineering, 2a. Edición, Prentice Hall Inc., Englewood Cliffs, NJ.

DDF (1993), "Normas técnicas complementarias", Diario Oficial de la Federación, Departamento del Distrito Federal, México, D.F.

Fajfar, P y P Gaspersic (1996), "The N2 method for the seismic damage analysis of RC buildings", Earthquake Engineering and Structural Dynamics, Vol. 25, pp 31-46.

Freeman, S A, J P Nicoletti y J V Tyrrell (1975), "Evaluation of existing buildings for seismic risk, A case study of Puget Sound Naval Shipyard, Bremerton, Washington", Memorias, First U.S. National Conference on Earthquake Engineering, EERI, Ann Arbor, Michigan, Estados Unidos, pp. 113-122.

Kilar, V y P Fajfar (1997), "Simple push-over analysis of asymmetric buildings", Earthquake Engineering and Structural Dynamics, Vol. 26, pp. 233-249.

Li, K N (1996), “CANNY-E. Three-dimensional nonlinear dynamic structural analysis. Computer program package”, Canny Consultants PTE. LTD, Singapur.

Luaces, F (1995) "Sobrerresistencia en estructuras a base de marcos de concreto reforzado", Tesis de Maestría, DEPFI, UNAM, México.

Moghadam, A S (1998), "Seismic torsional response of asymmetrical multi-storey frame buildings", Tesis doctoral, McMaster University, Hamilton, Ontario, Canadá.

Moghadam, A S y W K Tso (1996), "Damage assesssment of eccentric multistorey buildings using 3-D pushover analysis", Memorias, 11th World Conference on Earthquake Engineering (memorias en disco compacto), Acapulco, México.

Moghadam, A S y W K Tso (1997), "Seismic response of asymmetrical buildings using pushover analysis", en Seismic Design Methodologies for the Next Generation of Codes, P. Fajfar y H. Krawinkler (eds.), Balkema, Rotterdam, Holanda.

Moghadam, A S y W K Tso (1998), "Pushover analysis for asymmetrical multistorey buildings", Memorias, 6th.U.S. National Conference on Earthquake Engineering, EERI, Seattle, Washington, Estados Unidos.

Qi, X y J P Moehle (1991), "Displacement design approach for reinforced concrete structures Subjected to Earthquakes", Informe UCB/EERC-91/02, Earthquake Engineering Research Center, University of California, Berkeley, California.

Requena, M (1999), "Método aproximado para la evaluación del comportamiento sísmico de edificios altos”, Tesis de Maestría, DEPFI, UNAM, México. 